\title{
Diagenésis de la Formación Eagle Ford y sus marcadores térmicos como productora de gas no convencional
}

\author{
Diagenesis of the Eagle Ford Formation and its thermal markers as a producer of \\ unconventional gas
}

\author{
Aurea Yahaira González Betancourtt ${ }^{1,}$, Eduardo González Partida ${ }^{2}$, Noé Piedad Sánchez ${ }^{3}$, \\ Alejandro Carrillo Ghávez ${ }^{1}$, Luis Eduardo González Ruiz ${ }^{4}$, Daniel González Ruiz
}

${ }^{2}$ Centro de Geociencias, campus Juriquilla, Querétaro, Universidad Nacional Autónoma de México, Blvd. Juriquilla 3001, 76230, Querétaro, México.

${ }^{3}$ Innovación Tecnológica Aplicada a las Geociencias - Academia de Investigación A.C. Carboneras, Mineral de la Reforma, 42183, Hidalgo,México.

${ }^{4}$ Geologia Minería y Consultoría, S.A. de G.V. Ciprés 104 F, Frac. Jurica, 76100, Querétaro, México.

Centro Nacional de Investigaciones Avanzadas en Petrofísica CENIAPET, S. A. de G. V. Ciprés 104 F. Frac. Jurica, 76100, Querétaro, México.

* Autor para correspondencia: (A. González) aurea_1992@hotmail.com

\section{Cómo citar este artículo:}

González Betancourt, A. Y., González Partida, E., Piedad Sánchez, N., Carrillo Chávez, A., González Ruiz, L. E., González-Ruiz, D., 2020, Diagenésis de la Formación Eagle Ford y sus marcadorestérmicos como productora de gas no convencional: Boletín de la Sociedad Geológica Mexicana, 72 (2), A151219. http://dx.doi. org/10.18268/BSGM2020v72n2a151219

Manuscrito recibido: 13 de Mayo de 2019

Manuscrito corregido: 21 de Noviembre de 2019 Manuscrito aceptado: 13 de Diciembre de 2019

La revisión por pares es responsabilidad de la Universidad Nacional Autónoma de México.

\section{RESUMEN}

En México existen unidades geológicas compuestas por sedimentos de grano cfmuy fino y ricas en materia orgánica, que podrían considerarse como recursos potenciales de hidrocarburos. Entre ellas se encuentra la Formación Eagle Ford (Cenomaniano Tardío-Turoniano), la cual es el objeto de estudio en este trabajo. Dicha formación está localizada dentro de la Paleocuenca de Sabinas y sobre la Paleoplataforma Burro-Peyotes, en el estado de Coahuila. Anteriormente se ha definido que representa un sistema transgresivo depositado en un ambiente marino nerítico-medio con valores de carbón orgánico total (COT) entre 0.5 y $8 \%$ y con materia orgánica del tipo II predominante, por lo que podría considerarse como un posible recurso de hidrocarburos no convencional. Para ello se considera que un reservorio de lutitas gasíferas (shale gas) debe cumplir con ciertos requisitos, como: 1) Riqueza orgánica ( $>2 \%$ de COT), 2) tipo de kerógeno II o III, 3) valores de reflectancia de la vitrinita mayor a $1.2 \%$ Ro $(0.7 \%$ para shale oil $), 4)$ espesor mayor a $30 \mathrm{~m}$ y gran extensión, 5) fracturabilidad $(<40 \%$ de arcillas o ausencia de arcillas expandibles) o la presencia de un sistema de microfisuras, 6) sobrepresión, 7) profundidad adecuada, 8) heterogeneidad, entre otras. Con el objetivo de determinar el grado de madurez térmico de esta formación, se realizó un muestreo sistemático en afloramientos del estado de Coahuila para el análisis de estratigrafia a detalle (columnas CSI, CSII, CSV) y microtermometría de inclusiones fluidas. Los resultados ratifican que la Formación Eagle Ford es una secuencia sedimentaria rica en materia orgánica, compuesta localmente por lutita calcárea carbonosa con estratificación laminar que alterna a caliza arcillosa (mudstone-wackestone) y caliza clástica- bioclástica (packstone-grainstone). Respecto a las alteraciones diagenéticas, la porosidad de origen primario se ve afectada por los procesos de compactación y precipitación de cementos, también se generó una microporosidad secundaria debido al desarrollo de neomorfismo, dolomitización, fracturas y estilolitización. Además, se determinó que las temperaturas de homogeneización (Th) de inclusiones fluidas que alcanzó esta formación varían entre los $65^{\circ} \mathrm{C}$ y $125^{\circ} \mathrm{C}$, lo que la sitúa en la etapa catagenética de la generación de petróleo y gas húmedo. Asimismo, se observó que la evolución térmica de inclusiones fluidas en la cuenca no es homogénea.

Palabras clave: Recurso no convencional, petrografía, inclusiones fluidas, gas, diagénesis.

\section{ABSTRACT}

There are several organic matter-rich shale geologic formations in Mexico that could be considered non-conventional potential oil reservoirs; one of those formations is the Eagle Ford Formation (Late Cenomanian - Turonian), aim of this study. The Eagle Ford Formation is located within the Sabinas paleobasin and along the Burros-Peyote paleoplatform, in the Coahuila State. This formation has been previously described as a transgression system deposited in a shallow marine environment with a total organic carbon between 0.5 to $8 \%$ with type II organic matter. For these reasons, it could be considered a potential source for non-conventional hydrocarbons. Therefore, it is considered that a reservoir of shale gas must have certain requirements, such as: 1) high total organic carbon (> 2\% of COT), 2) presence of types II and III kerogen, 3) vitrinite reflectance values greater than $1.2 \%$ Ro (0.7\% for shale oil), 4) thickness greater than $30 \mathrm{~m}$ and large extension, 5) fracturability ( $<40 \%$ clays or absence of expandable clays) or the presence of a microcracking system, 6) overpressure, 7) adequate depth, 8) heterogeneity, and others. A systematic sampling was carried on outcrops of Coahuila State with the goal to determine the thermal maturity degree of the Eagle Ford Formation using a detailed stratigraphic analysis of three columns (CSI, CSII and $C S V$ ) and micro-thermometric of fluid inclusions. Our results indicate that the Eagle Ford Formation is a sedimentary sequence rich in organic matter, locally composed of carbonaceous carbonate shale in laminar stratification alternating with shaly limestone (mudstone-wackestone) and clastic o bioclastic limestone (packstone-grainstone). Regarding the diagenetic alteration, primary porosity is affected by compaction and cementation processes. However, it was also observed a secondary micro-porosity development due to neomorphism, dolomitization, fracturing and stylolitization processes. Measured homogenization temperatures (Th) in fluid inclusions are between $65^{\circ} \mathrm{C}$ and 125 ${ }^{\circ} \mathrm{C}$, reaching the temperature window to generate oil and humid gas. The thermal evolution through its facies changes in the basin is not homogeneous. Our results indicate that the Eagle Ford Formation is a sedimentary sequence rich in organic matter, locally composed of carbonaceous carbonate shale in laminar stratification alternating with shaly limestone (mudstone-wackestone) and clastic o bioclastic limestone (packstone-grainstone).

Keywords: Non-Conventional hydrocarbon reservoir, petrography, fluid inclusions, gas, diagenesis. 


\section{Introducción}

En la actualidad las metodologías disponibles para la reconstrucción de la historia térmica de una cuenca (como la de Sabinas) se pueden agrupar en cuatro categorías (Pagel et al., 2014): 1) los geotermómetros, 2) los cronómetros, 3) los crono-termómetros y 4) los geotermómetros cinéticos.

La historia térmica de las cuencas sedimentarias (y/o sus procesos diagenéticos) puede ser reconstruida, según Pagel et al., (2014), por diferentes marcadores: Los denominados inorgánicos (o minerales) y los orgánicos. Como es sabido, los procesos diagenéticos comprenden todas aquellas transformaciones físicas, químicas o biológicas que se producen dentro de una cuenca sedimentaria, inmediatamente después del depósito sedimentario, debido principalmente a: bioturbación, compactación, re-cristalización, disolución, reemplazamiento, autogénesis y cementación (Bathurst, 1975; Berner, 1980; Morton, 1985; Horsfield y Rullkötter, 1994; Morad et al., 2000; Buschaert et al., 2004; Pagel et al., 2014; González Ruiz et al., 2015; entre otros).

Todas las reacciones diagéneticas con procesos cinéticos variables están en función de la composición de los sedimentos, la presión, la temperatura, la composición y la naturaleza de los fluidos de poro, tamaño de los granos, porosidad, permeabilidad, volumen de fluidos, procesos de óxidoreducción, $\mathrm{pH}$ y tipo de materia orgánica, entre los más importantes (Chillingarian et al., 1992; Horsfield y Rullkötter, 1994; Budd et al., 1995).

La diagénesis, a su vez, se puede dividir en tres principales dominios (Morad et al., 2000; Worden y Burley, 2003; González Ruiz et al., 2015): 1) la diagénesis precoz o eogénesis, 2) la profunda o mesogénesis y 3) la tardía o telogénesis. La primera corresponde a todas aquellas transformaciones químicas, físicas y bioquímicas que se producen al momento del depósito de los sedimentos y su tiempo de enterramiento, cuando la química de los fluidos es controlada por el medio de depósito; aquí las aguas de los poros están en conexión con las superficiales.
El motor principal de la diagénesis precoz es la degradación de la materia orgánica que implica fuertes cambios "redox" y se da entre (Pagel et al., 2014) $30^{\circ} \mathrm{C}$ a $70{ }^{\circ} \mathrm{C}$, y entre 1 a $2 \mathrm{Km}$ de profundidad. La mesogénesis está relacionada con la interacción fluido-mineral, sin influencia de agentes superficiales; las transformaciones minerales son debido al enterramiento y al resultado de la interacción fluido/roca, y se tiene que considerar una evolución progresiva que dependa de las características mineralógicas, geoquímicas y petrofisicas de los depósitos sedimentarios y de su gradiente térmico.

La telogénesis está relacionada con la inversión estructural de la cuenca con infiltración de aguas meteóricas oxidantes en la zona de recarga, en los bordes de la cuenca que remplazan las aguas de poro de los sedimentos.

Es conveniente mencionar que la transformación de la materia orgánica es la única irreversible durante las tres etapas, realizando cambios durante la diagénesis con valores de temperatura ambiente $\left(30^{\circ} \mathrm{C} \sim\right)$ y $70^{\circ} \mathrm{C}+-10^{\circ}$, la catagénesis entre 70 y $160{ }^{\circ} \mathrm{C}+-20^{\circ}$ y la metagénesis entre 160 y $200{ }^{\circ} \mathrm{C}+-30{ }^{\circ} \mathrm{C}$. Estos valores también corresponden con el rango de temperaturas de las rocas sedimentarias, ya que, más allá de $200{ }^{\circ} \mathrm{C}$ aproximadamente, es cuando una roca sedimentaria entra a las facies metamórficas.

Por otro lado, un sistema petrolero que incluye elementos como la roca generadora, la roca almacén y la roca sello, así como procesos de generación, expulsión, migración, almacenamiento, preservación y degradación con una sincronía determinada (Magoon y Daw, 1994; Boyer et al., 2007), es producto de la procedencia de los sedimentos, del ambiente de depósito y de la evolución de los paleofluidos (Bathurst, 1975; Budd et al., 1995; Flügel, 2004; González-Partida et al., 2008a ;2008b). Tales procesos determinan la intensidad de los fenómenos diagenéticos (Bathurst, 1975; Chillingarian et al., 1992; Horsfield y Rullkötter, 1994; Budd et al., 1995; Flügel, 2004 y González Ruiz et al., 2015). De igual manera, se sabe que un yacimiento no convencional de hidrocarburos 


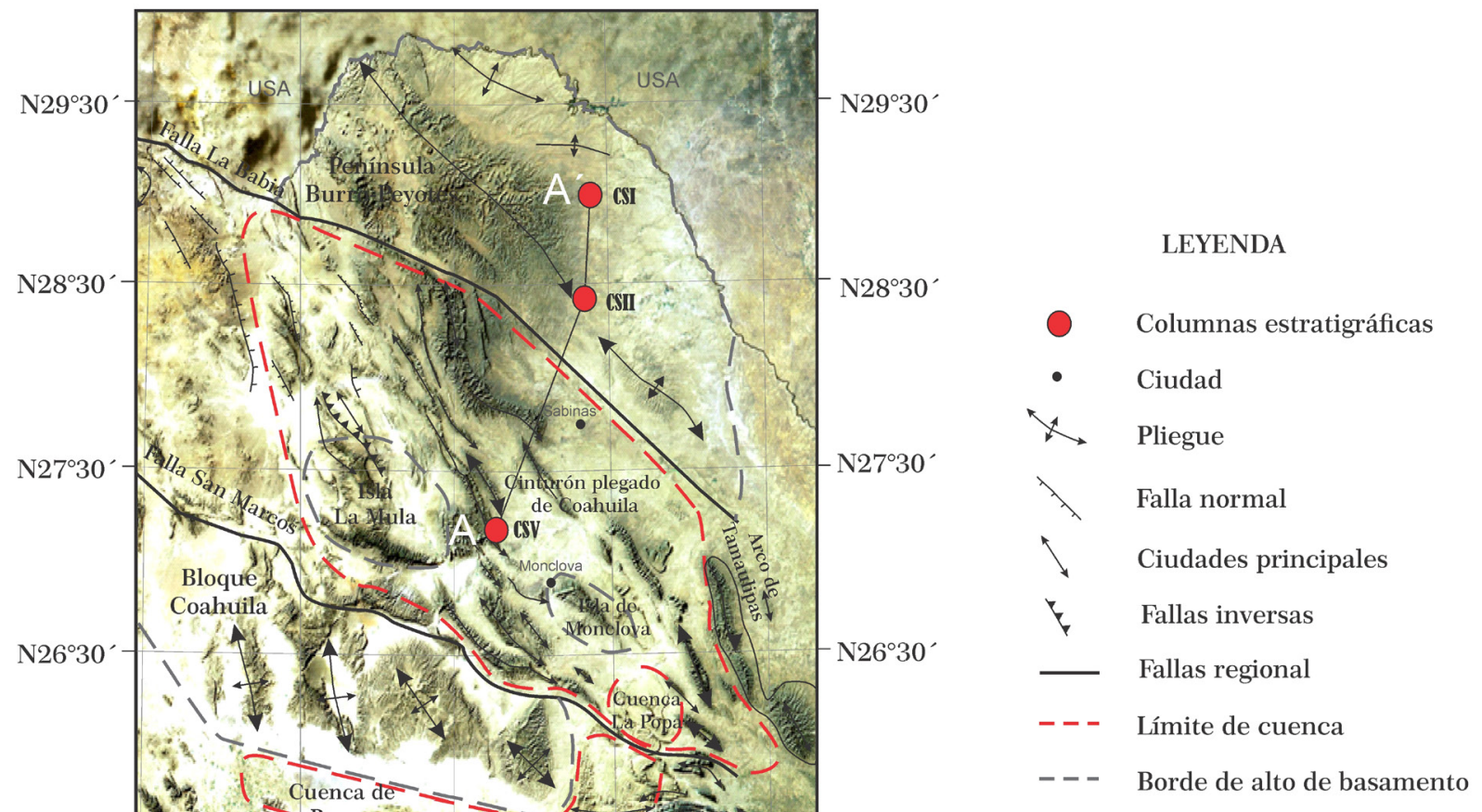

$-\mathrm{N} 25^{\circ} 30$
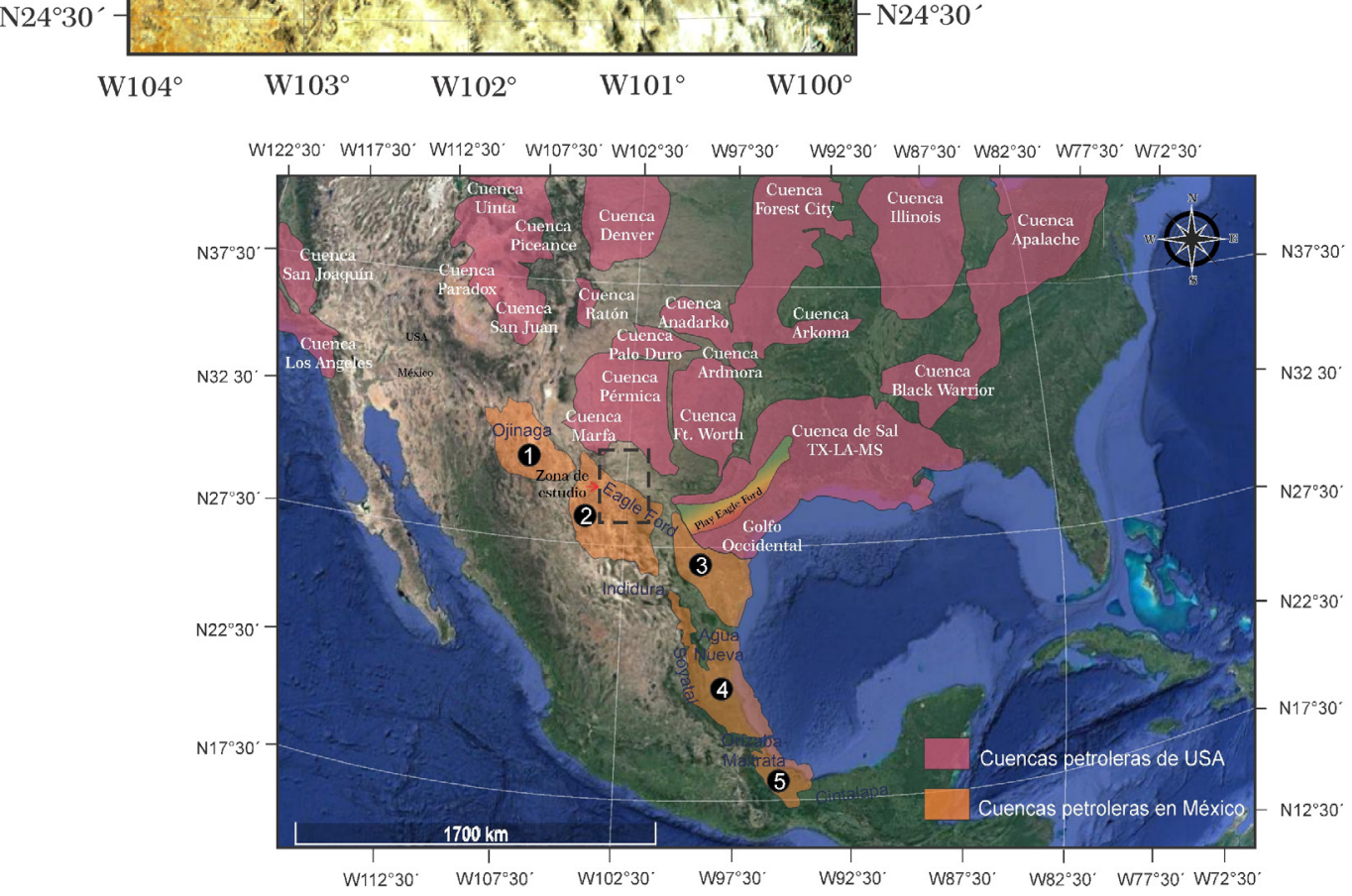

Figura 1 Localización de las columnas litológicas correspondientes a la Formación Eagle Ford que se estudiaron en este trabajo, y su posición con respecto a la Cuenca de Sabinas y estructuras geológicas sobresalientes; donde la columna CSV se encuentra sobre el cinturón plegado de Coahuila y las columnas CSI y CSII sobre la Península Burro-Peyotes (Transecto A- A'). Área de estudio ubicada en el mapa de cuencas productoras (imagen inferior), donde se muestra la distribución de cuencas productoras en Estado Unidos de América (USA), y las cinco principales cuencas con posible desarrollo de gas en lutitas en México según Petróleos Mexicanos (2011, 2012) y las Formaciones equivalentes a la Formación Eagle Ford. 
contrasta con un yacimiento convencional, ya que en realidad los fluidos atrapados en la roca generadora no fluyen con altas presiones, como lo harían en un yacimiento convencional, y es por ello que deben aumentar la permeabilidad e inyectar un gas o fluido a alta presión para que los hidrocarburos atrapados en ésta fluyan a través de los pozos horizontales. Sin embargo, si la roca generadora deja salir los hidrocarburos poco a poco a través de una permeabilidad inferior a 0.1 $m D$ (Lajous, 2015), es la causante de llenar todos los yacimientos convencionales. Aun así, la lutita puede funcionar al mismo tiempo como roca generadora y como roca almacén. Por lo anterior, las lutitas gasíferas pertenecientes a la Formación Eagle Ford se consideran como un recurso no convencional y son el objeto de este estudio. Las cualidades de las lutitas gasíferas propuestas por la United States Geological Survey (USGS) son: la inclusión de un alcance regional, la falta de rocas sello y trampas obvias, la ausencia de un contacto agua-gas bien definido, la presencia de fracturas naturales, una recuperación final estimada que, generalmente, es más baja que la de una acumulación convencional y una matriz de permeabilidad muy baja (Boyer et al., 2007; Askenazi et al., 2013; Santamaría-Orozco, 2000; Santamaría-Orozco y Horsfield, 2003; Santamaría-Orozco et al., 2009).

\section{Antecedentes}

Las cinco principales cuencas en México (Figura 1) con posible potencial de gas en lutitas son: 1) la Cuenca de Burgos (Formaciones Eagle Ford- Agua Nueva y Pimienta), 2) la Cuenca de Sabinas y Chihuahua (Formaciones Eagle Ford y La Casita), 3) Tampico (Formación Pimienta), 4) Tuxpan (Formaciones Pimienta y Tamaulipas) y 5) Veracruz (Formación Maltrata) con algunos horizontes ricos en materia orgánica del Jurásico Superior al Cretácico (Petróleos Mexicanos, 2011, 2012). Para el caso de la Guenca de Sabinas existen estudios de la materia orgánica de las Formaciones La Casita, Padilla y La Virgen que muestran una evolución térmica avanzada, donde el gas seco es termogénico, producto de materia orgánica tipo III con poco aporte del tipo II y una reflectancia de la vitrinita elevada (>2\% Ro) con profundidades de generación entre los 4 y 5 km (Piedad-Sánchez, 2004; Eguíluz de Antuñano, 2001; Martínez et al., 2015).

Por otro lado, la Formación Eagle Ford ha sido caracterizada como una roca generadora de hidrocarburos, junto con otras Formaciones como La Casita y La Peña (Santamaría- Orozco et al., 1991; Eguíluz de Antuñano, 2001; Eguíluz de Antuñano, 2007; González-Betancourt, 2018). Estas tres formaciones están conformadas por secuencias de rocas que se depositaron en ambientes marinos con aporte de terrígenos y materia orgánica predominantemente tipo II y poco del tipo III dentro de la Cuenca de Sabinas (Piedad-Sánchez, 2004; Martínez et al., 2015) y una madurez térmica muy elevada en rocas de la Formación La Casita del Jurásico Superior mayor de 2.5 \% Ro (Piedad-Sánchez, 2004; Martínez et al., 2015).

Además, Eguíluz de Antuñano (2001) propone un modelo térmico generalizado para la Cuenca de Sabinas con base en la profundidad y la edad de las Formaciones, en el que se plantea que la Formación Eagle Ford, en conjunto con la Formación Austin, ambas del Cretácico Superior, se encuentra en la etapa de generación principal de gas (Ewing, 2003) con un poder reflector de la vitrinita \% Ro entre 0.67 y 1.29 (Piedad- Sánchez, 2004).

Para la evolución térmica de la Cuenca de Sabinas se han utilizado diversas herramientas convencionales, como El estudio de la materia orgánica (Eguíluz de Antuñano, 2001; Eguíluz de Antuñano, 2007; Santamaría et al., 1991, 2009), estudios de isótopos estables y radioactivos (González-Sánchez et al., 2007), métodos que comprenden la reflectancia de la vitrinita (Piedad-Sánchez, 2004, 2005), estudios detallados de las arcillas y microtermometría de inclusiones fluidas en niveles estratigráficos más profundos en relación a la Formación Eagle Ford (González-Partida et 

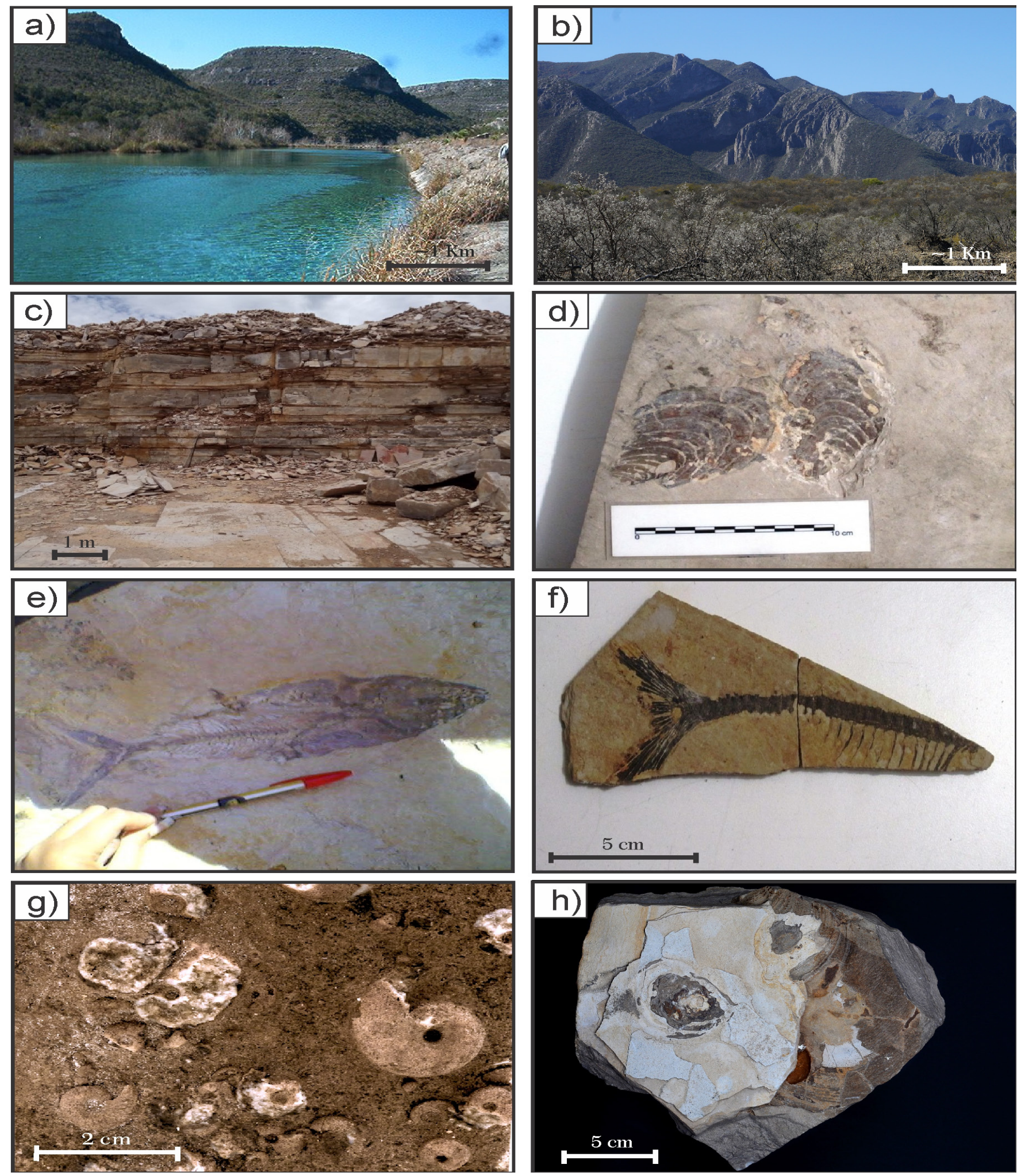

Figura 2 Afloramientos de la Formación Eagle Ford sobre la Península Burro-Peyotes (a), cerca de la localidad El Remolino, Coahuila, a 5 km de Piedras Negras (CSI) y dentro de la Cuenca de Sabinas (b), sobre la Sierra de Cristo al oeste de la localidad de San Antonio de la Cascada, Coahuila (CSV). (c) Formación Eagle Ford aflorando cerca de la zona de la columna CSII, donde se observan estratos delgados de lutita intercalados con caliza arcillosa. Esta formación se destaca por contener abundantes fósiles de peces (e,f), ammonites (g) y pelecípodos (d, h). 
al., 2008a, 2008b; González-Sánchez et al., 2007, 2009), etc. La presente investigación muestra resultados petrológicos y microtermométricos en la Formación Eagle Ford en tres columnas litológicas representativas (CSI, CSII y CSV) de la zona más profunda de la cuenca a la más somera, en un transecto que va del centro de la cuenca misma hacia la Península Burro -Peyotes (Sección A-'A, Figura 1).

\subsection{LA FORMAGIÓN EAGLE FORD Y SU DISTRI- BUCIÓN REGIONAL}

La distribución de la Formación Eagle Ford abarca la Cuenca de Sabinas, el Arco de Tamaulipas, el borde de la Cuenca de Burgos al norte de Nuevo León (Robeck et al., 1960; Eguíluz de Antuñano, 2001; Corona-Esquivel et al., 2006) y se extiende hacia el estado de Texas en Estados Unidos de
América, donde ha tenido una producción exitosa tanto de gas como de aceite (Santamaría-Orozco et al.,1990; Robinson, 1997; Padilla y Sánchez, 2016). El área de estudio comprende la Cuenca de Sabinas y la Península Burro-Peyotes, ubicadas en el estado de Coahuila, al noreste de la República Mexicana y al límite con la frontera de los Estados Unidos de América (Figura 1).

La Cuenca de Sabinas posee un área de 37000 $\mathrm{km}^{2}$ y su origen está relacionado con la apertura del Proto-Golfo de México (Padilla y Sánchez, 1986; Goldhammer et al., 1991, 1993; Goldhammer, 1999; Goldhammer y Johnson, 2001; Pindell, 1985, 1993; Pindell y Barrett, 1991; Pindell et al., 1988; Pindell y Kennan, 2009; Salvador, 1987, 1991a, 1991b, 1991c), y su "cierre tectónico" está relacionado con la Orogenia Laramide (Wilson, 1990; González-Sánchez et al., 2009). Antes del "cierre"

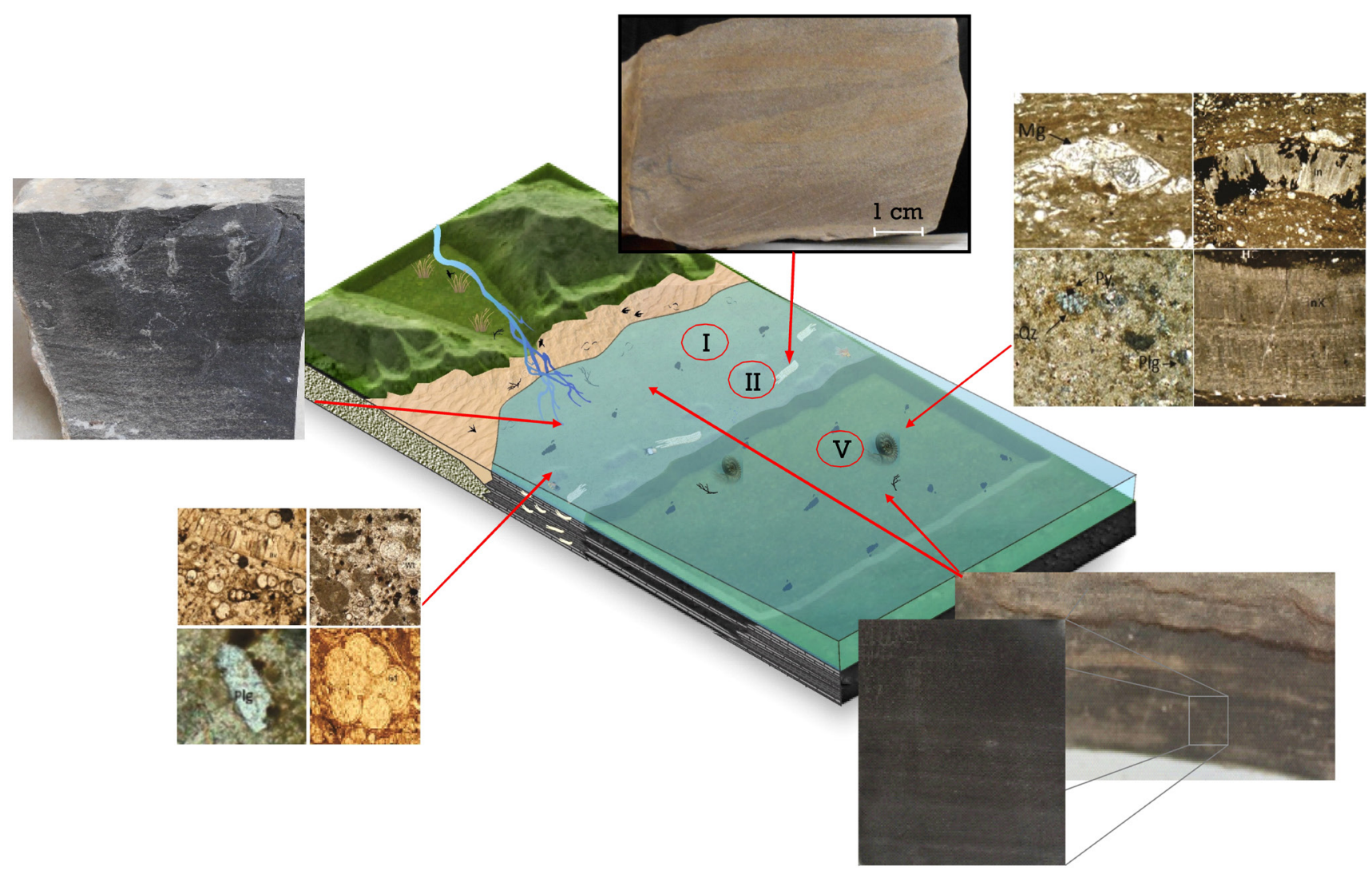

Figura 3 Ambiente de depósito de la Formación Eagle Ford en la parte norte de la Cuencas de Sabinas limitando con la Plataforma BurroPeyotes, correspondiente a una plataforma somera semi-protegida por bancos de arenas carbonatados y una cuenca con condiciones de baja energía y reducida en oxígeno. 
en el Cretácico Superior se depositaron sedimentos de ambiente marino y con aporte de terrígenos de la Formación Eagle Ford, lo que dio origen a una mezcla de materia orgánica tipo II (predominando este) y algo del tipo III (Piedad-Sánchez, 2004; Márquez, 1979; Longoria, 1984; SantamaríaOrozco et al., 1990; Martínez et al., 2015).

La Formación Eagle Ford fue descrita inicialmente por Roemer (1852) y luego por Shumard (1860), su localidad tipo está en Eagle Ford, condado de Dallas, Texas (EUA), donde se presenta como una lutita calcáreo-carbonosa con estratificación laminar, de color gris obscuro a negro al fresco, que alterna con cuerpos de mudstone (Figura 2). Otros autores la han descrito como una lutita negra intercalada rítmicamente con caliza arcillosa, clástica o bioclástica y arenisca cementada con carbonatos (Eguíluz de Antuñano, 2001; Sánchez et al., 2002;
Escalante et al., 2002; González-Betancourt, 2018). Se ha considerado que esta formación se depositó a finales del Cenomaniano y durante el Turoniano por su gran contenido de fauna estudiada (Moreman, 1927; Sellards, et al., 1966; Cobban 1988; Harries et al., 1996; Alvarado-Ortega et al., 2006), donde se destaca por contener abundantes fósiles de peces y conchas de amonitas y pelecípodos (Figura 2).

En la porción del sur de Texas la materia orgánica está representada por Exinitas: alginitas, esporinitas, cutinitas y resinitas; Vitrinitas: colinita y telinita, así como Inertinitas, (Robinson, 1997). Su espesor varía de 250 a 400 m (Eguíluz de Antuñano, 2001; EncisoCardenas, 2015) y se forma en un ambiente de depósito nerítico-medio que representa un sistema transgresivo (Figura 3), con facies de grano grueso y grano fino (MF1 y MF3), altamente compactadas, con pobre porosidad (Eguíluz de Antuñano, 2001;

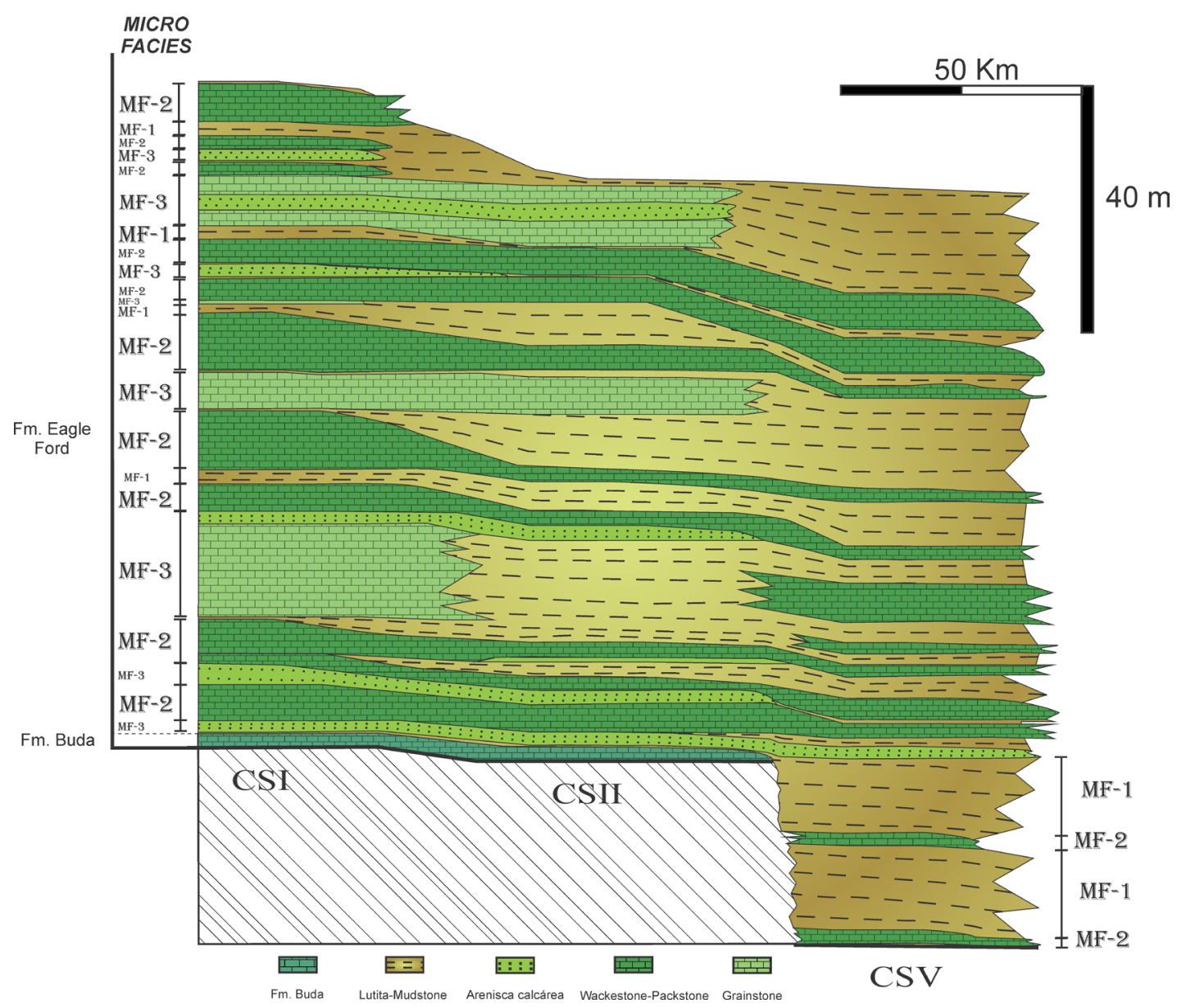

Figura 4 Estratigrafía de la Formación Eagle Ford con sus respectivos cambios de facies (MF-1, MF-2 y MF-3) desde la Plataforma Burro-Peyotes (CSI) hacia la Cuenca de Sabinas (CSV) en base a las descripciones petrográficas. Se observa un incremento en el contenido de arcillas y en el contenido de materia orgánica (MO) hacia la cuenca y un mayor contenido de caliza y arenisca con bajo contenido de MO sobre la plataforma. 
González-Betancourt, 2018) y alto contenido de carbono orgánico total (COT) entre 0.5 y 8\% del peso total de la roca (González y Holguín, 1992; Barboza et al., 1997; Santiago et al., 2000; Santiago et al., 2003; Pérez et al., 2000; Martínez et al., 2000; Romo et al., 2002; Escalante et al., 2002; Sánchez et al., 2002; Petróleos Mexicanos 1988, 2011, 2012), por lo que tiene una importancia petrolera como roca generadora (Santamaría et al.,1991). Se correlaciona en tiempo (Figura 1) con la Formación Agua Nueva de la Cuenca Tampico Misantla, Formación Indidura en la Sierra Madre Oriental y Formación Soyatal al oeste de la Cuenca Tampico Misantla, Formación Orizaba y Formación Maltrata de la Cuenca de Veracruz, en Chiapas con la Formación Cintalapa del Grupo Sierra Madre y en Chihuahua con la Formación Ojinaga (Santamaría- Orozco et al., 1991; Martínez et al., 2000; Eguíluz de Antuñano, 2001; Sánchez et al., 2002; Enciso- Cárdenas, 2015; entre otros).

\section{Metodología}

En el taller de laminación del Centro de Geociencias de la UNAM se cortaron 155 muestras de roca, formando láminas delgadas para la descripción petrográfica bajo microscopía de luz transmitida (Olympus BX60), usando la nomenclatura de Dunham (1962) y láminas doblemente pulidas para el análisis microtermométrico de inclusiones fluidas. La microtermometría se aplica en todo tipo de inclusiones fluidas: acuosas y de hidrocarburos (HC).

Para este estudio se usó una platina térmica tipo LINKAM, MDS600. Del análisis microtermométrico se obtienen tres tipos de información: 1) $\mathrm{Te}=$ temperatura eutéctica del sistema, 2$) \mathrm{Tf}=$ temperatura de fusión del último cristal de hielo dentro de la inclusión, y 3) Th = temperatura de homogeneización de fases. Generalmente, en inclusiones fluidas acuosas se observan estos tres parámetros, pero en las de $\mathrm{HC}$, por lo general, solo se puede medir la Th. El análisis se realiza con la ayuda de un microscopio petrográfico de luz transmitida, de 1000 aumentos, al que se le ha adicionado una platina térmica que permite el calentamiento de especimenes hasta los $+650^{\circ} \mathrm{C}$ y un sistema de enfriamiento a base de nitrógeno líquido que baja la temperatura hasta $-200^{\circ} \mathrm{C}$.

$\mathrm{El}$ primer paso (petrografía de inclusiones fluidas) consiste en reconocer los diferentes tipos de inclusiones y hacer una descripción detallada de las mismas, incluyendo su forma, tamaño y fases presentes, que pueden ser sólida, líquida o gaseosa. El tamaño pequeño de las inclusiones fluidas, que varía de 5 a 30 micras $(\mu)$, es lo que hace que esta técnica sea en la actualidad difícil y que requiera de herramientas especializadas para su operación. En esta etapa se debe de tomar en cuenta (por medio de la observación petrográfica) la posible existencia de fenómenos perturbadores como: encuellamiento, escape y relleno de fluido (leakage), metaestabilidad, agrandamiento (stretching) y decrepitación.

La etapa más importante tiene lugar en la microtermometría, técnica que consiste en realizar medidas a bajas y altas temperaturas. El método radica en provocar la solidificación (bajo observación microscópica) de las fases fluidas mediante la disminución progresiva de la temperatura (hasta $-200^{\circ} \mathrm{G}$ ). El punto de solidificación de una sustancia está en función de su contenido salino, por lo cual esta determinación permite el cálculo del porcentaje de sales disueltas en la fase acuosa en fluidos subsaturados.

El proceso de calentamiento se realiza sobre las mismas inclusiones, inmediatamente después de analizarlas a baja temperatura. Ésta se va aumentando a una tasa de calentamiento lenta para permitir el equilibrio de las fases y poder detectar los fenómenos de fusión y homogeneización $\left(1^{\circ} \mathrm{C}\right.$ por minuto). En medios diagenéticos, es recomendable primero calentarlas y después enfriarlas para evitar que se produzca una decrepitación, sobre todo en fluidos muy densos.

\subsection{EL MUESTREO}

Se realizaron expediciones al estado de Coahuila para el levantamiento de tres columnas estratigráficas: 1) CSI, 2) CSII y 3) CSV, ubicadas, 

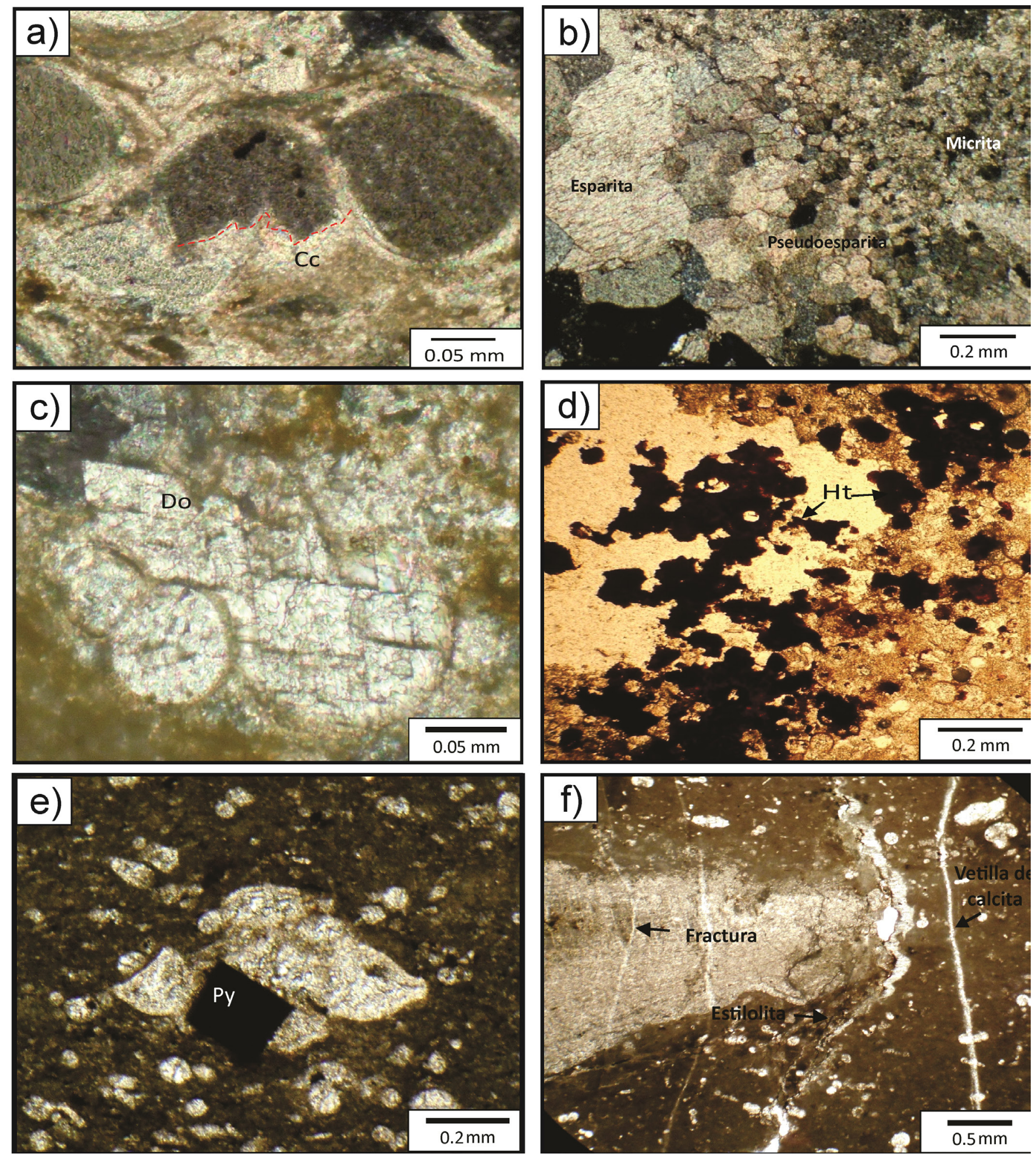

Figura 5 Ejemplos dealteraciones diagenéticas observadas bajoel microscopio petrográfico;a)Granos cortados y generación decemento de calcita(Cc);b)Neomorfismoagradacional;c)Dolomita(Do)dentrodeorganismosfósilesychertintrapartícula;d) Disolución degranosycemento dehematita(Ht);e)Compactación degranos, seobservancristales depirita(Py) presentes;f)Estilolitas, fracturasyvetillas decalcita presentes. 
respectivamente, en 1) Localidad El Remolino, Coahuila, a $50 \mathrm{~km}$ de Piedras Negras, coordenadas UTM 14R0298723/ 3187001, 2) Rancho La Mota, $30 \mathrm{~km}$ al oeste del municipio de Morelos, coordenadas UTM 14R0286630/ 3143773, y 3$)$ Sierra de Cristo al oeste de la localidad de San Antonio de la Cascada, coordenadas UTM 14R0225163/ 3013576 (Figura 1).

Los levantamientos estratigráficos se realizaron tomando una muestra de roca cada 2 metros, distancia medida perpendicularmente al echado de los estratos, obteniendo así espesores entre 90 y 125 $\mathrm{m}$. Para hacer los estudios microtermométricos es necesario realizar otros previos sobre petrología convencional, la cual debe establecer una secuencia paragenética con una identificación del tiempo relativo de formación de eventos y micro-eventos, de tal manera que se puedan elegir las muestras de acuerdo con el evento que se pretende estudiar.

\section{Resultados}

\subsection{PETRografíA}

La Formación Eagle Ford presenta un cambio de facies desde la Península Burro-Peyotes hacia la Cuenca de Sabinas (Figura 4). Sobre la península se realizaron dos levantamientos litológicos que corresponden a las columnas I y II, con 110 y 90 $\mathrm{m}$ de espesor, respectivamente. Las microfacies dominantes son MF-2 y MF-3 (González-Betancourt, 2018).

En éstas se pueden observar estratos de wackestone y packstone de tonalidades grises y espesores entre 1 y 20 cm (MF-2), además de grainstone y arenisca cementada con carbonatos, compuestas de fragmentos calcáreos, bioclastos y extraclastos de cuarzo y feldespatos, así como también material fecal (MF-3). Éstos se DOIn intercalados por estratos delgados de lutita de color café amarillento y espesores entre 1 y $30 \mathrm{~cm}$ (MF-1).

El depósito está acomodado en estratos casi horizontales con rumbo NNW-SSE y echados entre $5^{\circ}-20^{\circ} \mathrm{NE}$ al norte de la península y
NNE- SSW con echados cercano a $10^{\circ} \mathrm{SE}$ en la parte sur de la península. A la base de la secuencia la estratificación y laminación es paralela, sobreyacida por estratificación ondulada, cruzada, anastomosada y en ocasiones tipo flaser. Las estructuras principales consisten en rizaduras con crestas onduladas, estructuras de carga, diques sedimentarios y deformación convoluta. Los organismos fósiles observados son principalmente ammonites, bivalvos, pelecípodos (Inoceramus labiatus) y bioclastos, así como impresiones de ramas, icnofósiles, galerías y materia orgánica. Los microfósiles más abundantes son foraminíferos planctónicos de formas pequeñas, globulosas, biseriadas, planoespiraladas o troncoespiraladas, calciesférulas, peloides, en menor proporción radiolarios y foraminíferos bentónicos.

La alteración diagenética de la roca es mínima y consiste de fracturamiento, oxidación, disolución y desarrollo de caliche a la cima de los afloramientos. En lámina delgada, se observa la siguiente secuencia paragenética; durante la diagenésis precoz o eogénesis se observa la precipitación de cristales de pirita, cemento de calcita intergranular y de hematita en los horizontes superiores, además de que la roca presenta un grado de compactación de bajo a moderado. Para la etapa mesogenética se observa disolución en organismos fósiles, desarrollo de estilolitas, precipitación de cemento de calcita intragranular, neomorfismo agradacional y dolomitización. Finalmente se observan vetillas de calcita y fracturas casi perpendiculares a la estratificación (Figura 5). En el interior de la Cuenca de Sabinas los estratos estudiados se encuentran fuertemente plegados. La secuencia en esta área es de 125 m de espesor y consiste de las microfacies denominadas MF-1 y MF-2 (Eguíluz de Antuñano, 1984; González-Betancourt, 2018). Está constituida por lutitas, limolitas y mudstone (MF-1) e intercalados por wackestone (MF-2) (Figuras 4 y 7). Las estructuras sedimentarias primarias consisten de estratificación y laminación paralela u ondulada sin bioturbación.

El contenido fosilífero es menos variado que sobre la península, pero a nivel microscópico 
se observa un gran contenido de foraminíferos planctónicos de mayor tamaño y formas más ornamentadas y carenadas, abundantes filamentos, algunos bioclastos y paredes de diferentes tipos de Inoceramus con paredes aragoníticas aún preservadas.

La secuencia paragenética de los procesos de alteración que se observan inicialmente, seguidos al depósito de los sedimentos y durante la eogénesis, son compactación y precipitación de cementos de carbonato, los cuales afectaron la porosidad primaria, así como también la generación de cristales de pirita típica de ambientes reducidos en oxígeno. Durante la etapa de mesogénesis se observa la precipitación de cemento de sílice (chert o calcedonia) es los espacios porosos de la roca, pero también tenemos evidencia de disolución en organismos fósiles y en minerales inestables, como feldespatos y líticos volcánicos, el desarrollo de estilolitización moderada a intensa, neomorfismo y dolomitización, los cuales generaron una porosidad secundaria en la roca. Esto como consecuencia de la interacción de los fluidos provenientes del centro de la cuenca con las rocas de la Formación Eagle Ford. Para la etapa de telogénesis tenemos la fracturación natural de la roca, generación de vetillas de calcita y microplegamiento, además de una segunda precipitación de cemento carbonatado intragranular (dentro de fósiles que presentaban disolución y de granos cortados), que pudo haberse desarrollado también durante la etapa mesogenética.

El contenido de COT reportado en Estados Unidos va entre 4.5 y $5.5 \%$ (Askenazi et al., 2013), mientras que en México se ha reportado
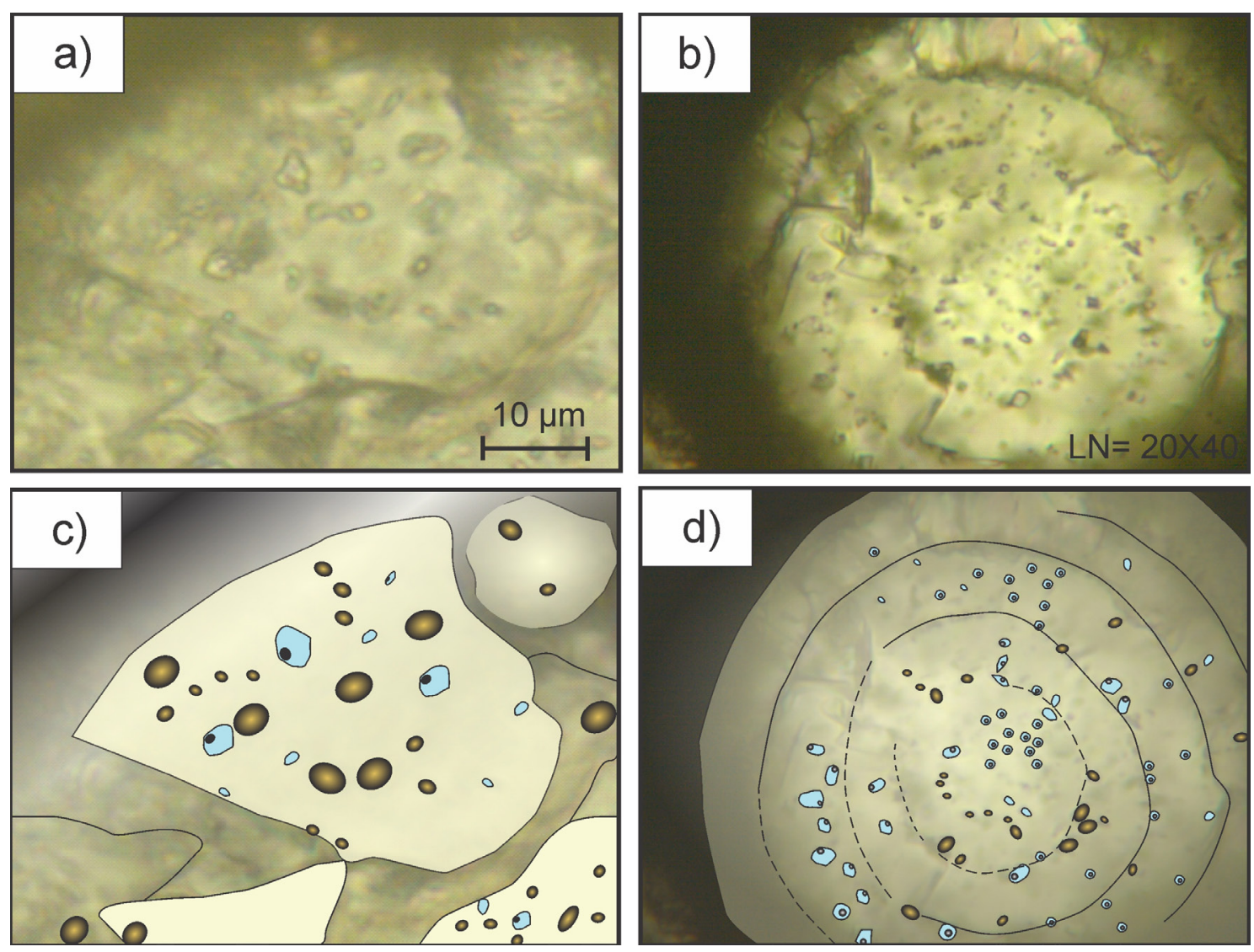

Figura 6 Paragénesis de inclusiones fluidas monofásicas y bifásicas de hidrocarburos y tipo acuoso, dentro de cristales de calcita (a y c) y organismos fósiles recristalizados (b y d). 
una gran variación de valores del COT; teniendo en el noreste de la península Burro-Peyotes un valor COT de entre 3 y 4\% cerca de la ciudad de Piedras Negras (próximo a la columna CSI), entre 2.5 y $4.7 \% \quad 10 \mathrm{Km}$ al noroeste de Villa Hidalgo (más próximo a la columna CSII), para el noroeste de la Cuenca de Burgos los valores están entre 1.95 y $2.71 \%$ (Martínez- Contreras,
2015; Petróleos Mexicanos, 2011; 2012) y en el interior de la Cuenca de Sabinas los valores de COT están entre 2 y 4\%, y >4\% (Petróleos Mexicanos, 2012) cercano al área de Monclova (próximo a la columna CSV). De manera general en la Formación Eagle Ford los contenidos de carbono orgánico total (COT) son altos, estando entre el 0.5 y $8 \%$ del peso total de la roca, donde

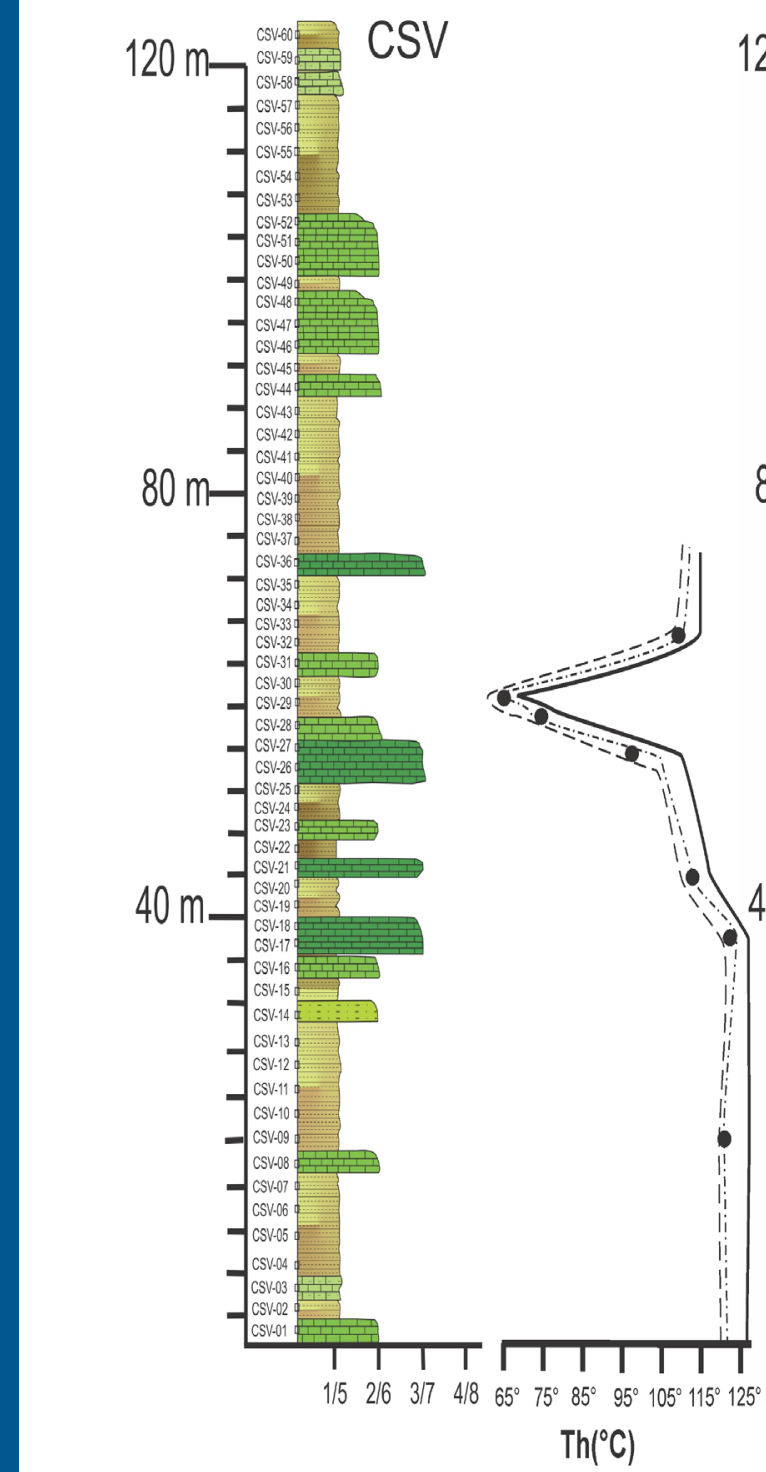

$\mathrm{Th}\left({ }^{\circ} \mathrm{C}\right)$

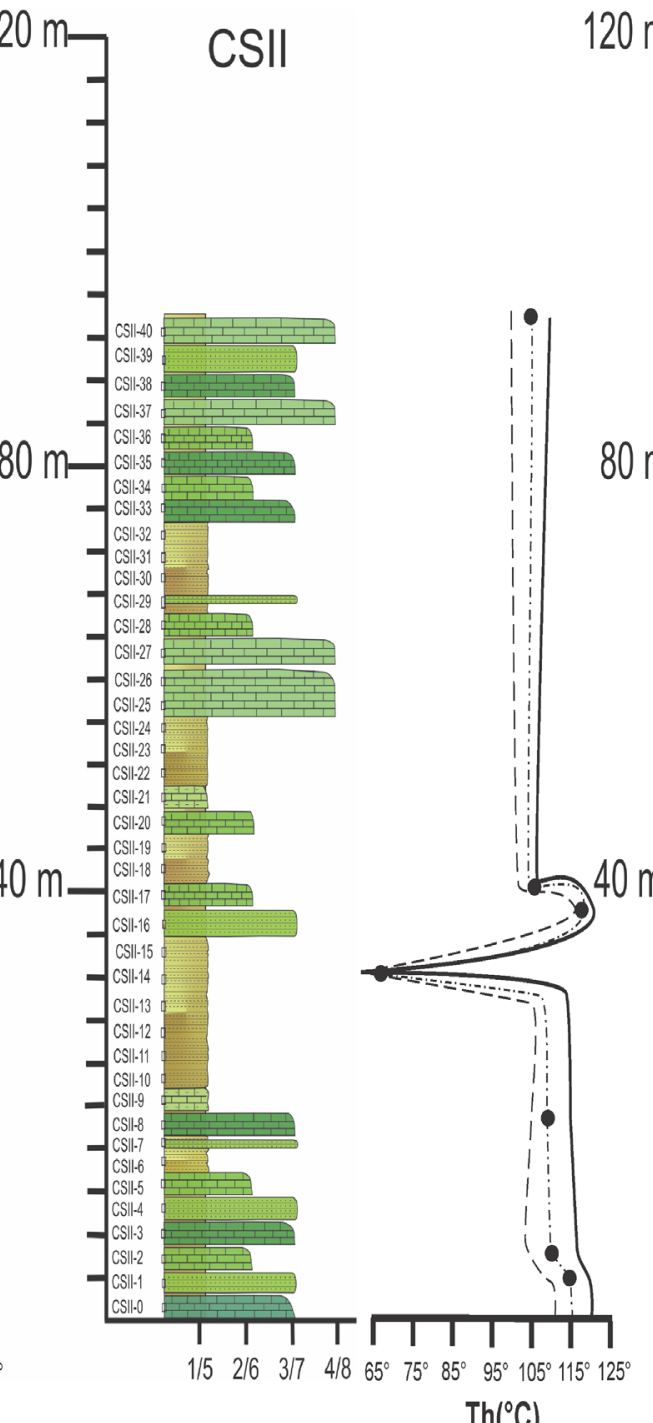

$\mathrm{Th}\left({ }^{\circ} \mathrm{C}\right)$

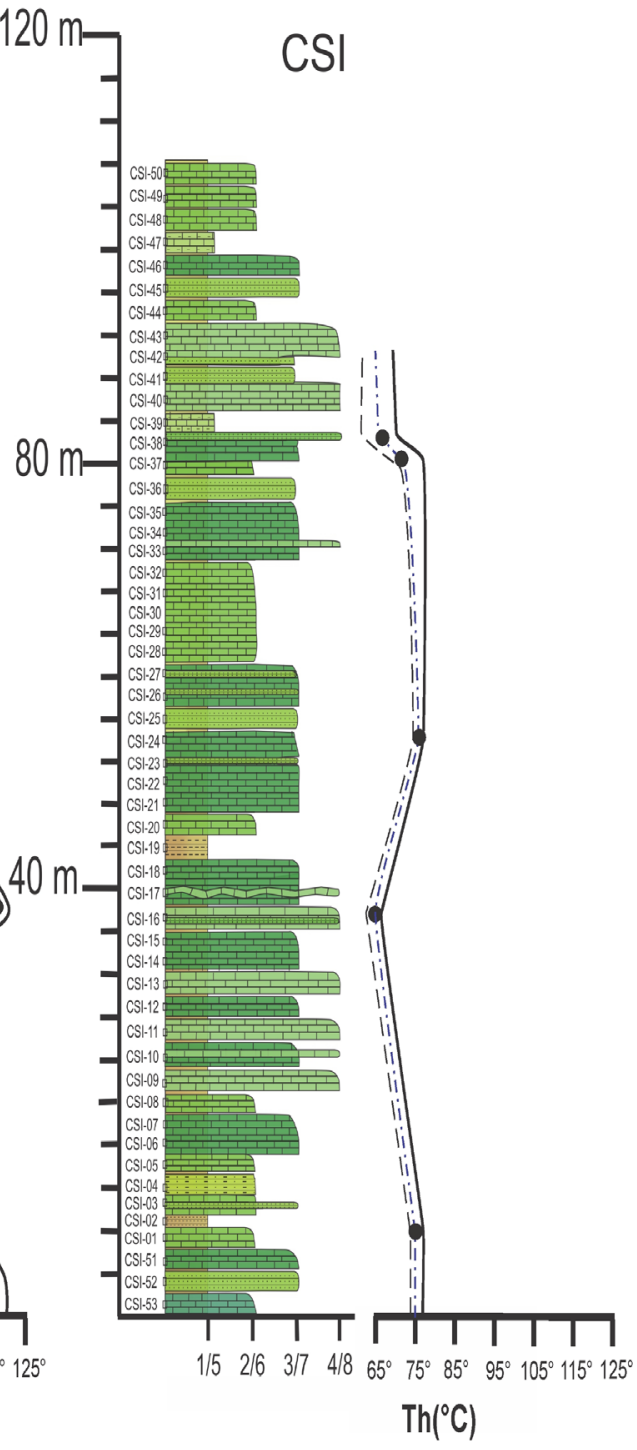

Análisis de mínima promedio máxima

\begin{abstract}
inclusiones fluidas
\end{abstract}
$---$

Figura 7 Estratigrafía de la Formación Eagle Ford con los resultados del análisis microtermométrico en las inclusiones fluidas de las tres columnas estudiadas, desde la Cuenca de Sabinas hacia la Plataforma Burro-Peyotes. Se presentan las muestras analizadas con su Th mínima, máxima y promedio, observando la variación perpendicular a la estratigrafía. Donde Th = temperatura de homogenización. 
Tabla 1. Resumen de los datos microtermométricos de inclusiones fluidas de rocas de la Formación Eagle Ford. Las muestras corresponden a lutitas generadoras de hidrocarburos en la Cuenca de Sabinas. También se anexan datos del poder reflector de la vitrinita (\%Ro) de muestras cercanas al área donde se recolectaron las columnas CSI, CSII y CSV.

\begin{tabular}{|c|c|c|c|c|c|c|c|c|c|c|c|c|}
\hline $\begin{array}{l}\text { Tipo de } \\
\text { depósito }\end{array}$ & $\begin{array}{c}\text { Nombre } \\
\text { del } \\
\text { depósito }\end{array}$ & $\begin{array}{c}\text { Información } \\
\text { adicional }\end{array}$ & $\begin{array}{c}\text { Columna } \\
\text { estratigráfica }\end{array}$ & $\begin{array}{l}\text { Clave } \\
\text { de } \\
\text { muestra }\end{array}$ & Mineral & n & $\begin{array}{l}\text { Tipo de } \\
\text { inclusión } \\
\text { fluida }\end{array}$ & $\begin{array}{c}\text { Rango } \\
\text { de Th } \\
\left({ }^{\circ} \mathrm{C}\right)\end{array}$ & $\begin{array}{c}\text { Th } \\
\text { Promedio } \\
\left({ }^{\circ} \mathrm{C}\right)\end{array}$ & $\begin{array}{l}\text { Rango de } \\
\text { salinidad } \\
\text { (wt.\% } \\
\mathrm{NaCl} \\
\text { equiv.) }\end{array}$ & $\begin{array}{c}\text { Salinidad } \\
\text { promedio } \\
\text { (wt.\% } \\
\mathrm{NaCl} \\
\text { equiv.) }\end{array}$ & $\begin{array}{c}\text { \%Ro } \\
\text { (Piedad } \\
\text { Sánchez, } \\
\text { 2004; } \\
\text { PEMEX, } \\
\text { 2012) }\end{array}$ \\
\hline \multirow{19}{*}{$\begin{array}{l}\text { Lutita } \\
\text { gasífera }\end{array}$} & \multirow{19}{*}{$\begin{array}{l}\text { Formación } \\
\text { Eagle Ford } \\
\text { (Cenomani } \\
\text { ano tardío- } \\
\text { Turoniano) }\end{array}$} & \multirow{19}{*}{$\begin{array}{l}\text { Ricas en } \\
\text { materia } \\
\text { orgánica de } \\
\text { tipo II y III. } \\
\text { Roca } \\
\text { moderada a } \\
\text { altamente } \\
\text { compactada } \\
\text { y baja } \\
\text { porosidad. }\end{array}$} & I & CSI-1 & Calcita & 26 & $\mathrm{~L}+\mathrm{V}$ & $73-76$ & 74.69 & $8.3-8.3$ & 8.3 & 0.5 \\
\hline & & & I & CSI-16 & Calcita & 21 & $\mathrm{~L}+\mathrm{V}$ & $63-66$ & 64.48 & $8.1-8.1$ & 8.1 & 0.5 \\
\hline & & & I & CSI-24 & Calcita & 28 & $\mathrm{~L}+\mathrm{V}$ & $74-77$ & 75.64 & $8.0-8.0$ & 8.0 & 0.5 \\
\hline & & & I & CSI-37 & Calcita & 15 & $\mathrm{~L}+\mathrm{V}$ & $70-77$ & 72.40 & 7.9-7.9 & 7.9 & 0.5 \\
\hline & & & I & CSI-38 & Calcita & 17 & $\mathrm{~L}+\mathrm{V}$ & 61-69 & 65.94 & $7.6-7.6$ & 7.6 & 0.5 \\
\hline & & & II & CSII-1 & Calcita & 29 & $\mathrm{~L}+\mathrm{V}$ & $110-120$ & 115.90 & $7.2-7.2$ & 7.2 & 1.29 \\
\hline & & & II & CSII-2 & Calcita & 25 & $\mathrm{~L}+\mathrm{V}$ & $103-117$ & 110.04 & $9.2-9.2$ & 9.2 & 1.29 \\
\hline & & & II & CSII-8 & Calcita & 28 & $\mathrm{~L}+\mathrm{V}$ & $105-115$ & 109.75 & $8.7-8.7$ & 8.7 & 1.29 \\
\hline & & & II & CSII-14 & Calcita & 21 & $\mathrm{~L}+\mathrm{V}$ & $30-36$ & 33.71 & N/A & N/A & 0.6 \\
\hline & & & II & CSII-16 & Calcita & 13 & $\mathrm{~L}+\mathrm{V}$ & $116-120$ & 118.15 & $5.9-5.9$ & 5.9 & 1.29 \\
\hline & & & II & CSII-17 & Calcita & 18 & $\mathrm{~L}+\mathrm{V}$ & $101-106$ & 104.17 & 7.9-7.9 & 7.9 & 1.29 \\
\hline & & & II & CSII-40 & Calcita & 38 & $\mathrm{~L}+\mathrm{V}$ & $100-110$ & 104.61 & $12.8-12.8$ & 12.8 & 1.29 \\
\hline & & & V & CSV-29 & Calcita & 39 & $\mathrm{~L}+\mathrm{V}$ & $67-77$ & 72.54 & $5.0-5.0$ & 5.0 & 0.8 \\
\hline & & & V & CSV-30 & Calcita & 34 & $\mathrm{~L}+\mathrm{V}$ & 63-68 & 65.18 & $6.4-6.4$ & 6.4 & 0.67 \\
\hline & & & V & CSV-9 & Calcita & 36 & $\mathrm{~L}+\mathrm{V}$ & $120-126$ & 121.89 & $9.2-9.2$ & 9.2 & 1.88 \\
\hline & & & V & CSV-18 & Calcita & 13 & $\mathrm{~L}+\mathrm{V}$ & $121-126$ & 124.38 & $8.3-8.3$ & 8.3 & 1.88 \\
\hline & & & V & CSV-21 & Calcita & 22 & $\mathrm{~L}+\mathrm{V}$ & $110-117$ & 113.23 & 6.4-6.4 & 6.4 & 1.88 \\
\hline & & & V & CSV-27 & Calcita & 26 & $\mathrm{~L}+\mathrm{V}$ & $104-110$ & 105.69 & $5.0-5.0$ & 5.0 & 1.80 \\
\hline & & & V & CSV-33 & Calcita & 22 & $\mathrm{~L}+\mathrm{V}$ & $109-115$ & 110.73 & 7.9-7.9 & 7.9 & 1.88 \\
\hline
\end{tabular}

el miembro inferior o más arcilloso de la Formación Eagle Ford es más rico en el contenido de COT (González y Holguín, 1992; Barboza et al., 1997; Santiago et al., 2000; Pérez et al., 2000; Martínez et al., 2000; Romo et al., 2002; Escalante et al., 2002; Sánchez et al., 2002; Santiago et al., 2003, Petróleos mexicanos 1988, 2011, 2012).

\subsection{MICROTERMOMETRÍA DE INGLUSIONES FLUIDAS}

Las inclusiones fluidas, objeto del estudio microtermométrico en calcita y dolomita de las tres columnas de la Formación Eagle Ford, fueron primarias de acuerdo a los criterios de identificación enunciados por Roedder (1984), Goldstein y Reynolds (1994), Boiron y Dubessy (1994) y Goldstein (2003). En la descripción petrográfica se reconocieron tres tipos de inclusiones; 1) monofásicas de líquido (L), 2) bifásicas de líquido+ vapor $(\mathrm{L}+\mathrm{V})$ compuestas de salmueras y 3) bifásicas de hidrocarburos. Estas inclusiones se observan atrapadas en organismos recristalizados, cementos sobre granos cortados o cementos en las cavidades de la roca. El tamaño de las inclusiones fluidas va entre $2 \mu \mathrm{m}$ a $15 \mu \mathrm{m}$ de largo, presentando formas redondeadas, de cuadrilateros y/o anhedrales (Figura 6).

El grado de relleno por estimación visual es de 0.95, teniendo una fase líquida dominante. Las inclusiones de hidrocarburos se observan de color café obscuro y corresponden a metano; las inclusiones de salmueras se observan transparentes en ocasiones con inclusiones sólidas de cristales heredados de hematita, de color café-rojizo. Las inclusiones pueden ser encontradas inmersas al azar sobre cemento de calcita que remplaza a granos cortados o en cristales de dolomita intracristalina y dentro de organismos fósiles reemplazados, por lo que la mayoría de ellas representan la fase de mayor soterramiento de las rocas o mesogénesis.

Para el análisis microtermométrico sólo se analizaron las inclusiones bifásicas acuosas, de las cuales se obtuvieron las temperaturas de homogeneización (Th), y las temperaturas de fusión final (Tff), con lo que se calculó el porcentaje de salinidad (Wt \% $\mathrm{NaCl} \mathrm{Eq.).} \mathrm{Los} \mathrm{resultados} \mathrm{se} \mathrm{presentan} \mathrm{en} \mathrm{la} \mathrm{tabla}$ $1 \mathrm{y}$ en la figura 7. 
Del análisis de la información obtenida se observa que sobre la Península Burro-Peyotes las temperaturas de homogeneización promedio (columna I) son de $\mathrm{Th}=70.6^{\circ} \mathrm{C}$ con salinidades promedio de $8 \mathrm{wt} \% \mathrm{NaCl}$ Eq., la columna II presenta una Th mayor de $99.5^{\circ} \mathrm{C}$, así como una salinidad promedio de $8.62 \mathrm{wt} \% \mathrm{NaCl}$ Eq., mientras que en el interior de la Cuenca de Sabinas la columna V tiene una temperatura de homogeneización promedio de $\mathrm{Th}=101.95^{\circ} \mathrm{C}$ y una salinidad de $6.9 \mathrm{wt} \% \mathrm{NaCl}$ Eq. Comparando los datos de las tres columnas, las Th mayores se encuentran en el interior de la Cuenca de Sabinas y en el contacto con el borde de la Península Burro-Peyotes (Figura 8), lo que probablemente esté asociándo principalmente al proceso de soterramiento de la Formación Eagle
Ford y a una maduración diferencial de la materia orgánica a lo largo de la paleogeografía.

\section{Discusión}

Con base en los análisis petrográficos se observa que la Formación Eagle Ford presenta un cambio de facies desde la Península Burro-Peyotes hacia la Cuenca de Sabinas, donde el contenido de arcillas aumenta. Sobre la Península predominan las lutitas ricas en materia orgánica, intercaladas con calcarenitas y caliza (mudstone-grainstone), presentando abundante contenido fósil y dominado por foraminíferos planctónicos de formas pequeñas, globulosas, biseriadas, planoespiraladas

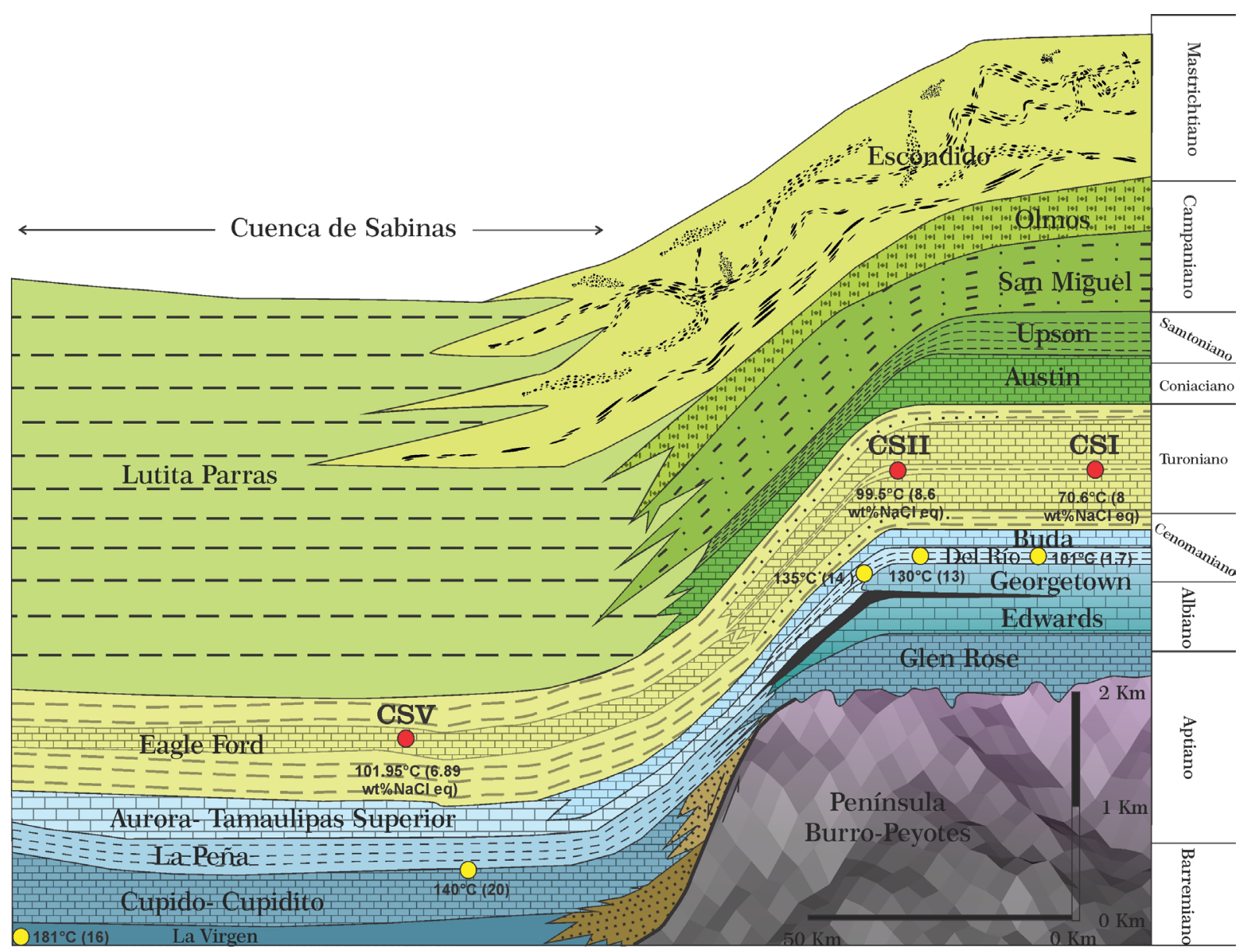

Figura 8 Corte que muestra la distribución térmica y salinidades en porcentaje de las muestras analizadas a lo largo de Cuenca de Sabinas. Los puntos rojos corresponden a las Th y salinidad promedio de la Formación Eagle Ford (CSI, CSII, CSV), mientras que los puntos amarillos corresponden a los yacimientos tipo Mississippi Valley medidos por González- Sánchez et al., (2007). Donde Th= Temperatura de homogenización. 
o troncoespiraladas y bivalvos, siendo ésta área interpretada como una plataforma somera protegida por bancos de arenas carbonatados, que permitieron el depósito de las arcillas y las partículas de materia orgánica en un ambiente reducido en oxígeno, pero que temporalmente se vio afectada con periodos de tormentas y oleaje, que depositaron las calcarenitas con estratificación cruzada y anastomosada y los cementos o cristales de hematita. Por consiguiente, las areniscas suelen tener menor contenido de materia orgánica en comparación con las lutitas.

En el interior de la Cuenca de Sabinas encontramos depósitos con grandes espesores de lutita de color gris obscuro, un alto contenido de materia orgánica, filamentos y laminación muy fina y paralela sin perturbación, intercalada por calizas (mudstone- wackestone) y con presencia de foraminíferos planctónicos de formas más grandes, ornamentadas y carenadas que ocuparon los nichos más profundos (Molina, 2004) Dicha secuencia fue depositada en aguas tranquilas con condiciones anóxicas necesarias para la preservación de la materia orgánica, evidenciado por las concentraciones de filamentos, el color obscuro de las rocas y la presencia de pirita framboidal en las rocas.

En la Cuenca de Sabinas existen estudios previos de la materia orgánica de las Formaciones La Casita, Padilla, La Virgen, La Peña, Eagle Ford y Olmos, que muestran una evolución térmica predominantemente avanzada, donde el gas seco es termogénico, producto de materia orgánica tipo III con aporte del tipo II, y una reflectancia elevada de la vitrinita a profundidad, por lo que se establecen profundidades de generación de gas entre los 3 y 4 km (Piedad-Sánchez, 2004; Martínez et al., 2015; Eguíluz de Antuniano et al., 2001). Por lo que la Formación Eagle Ford ha sido caracterizada como una roca generadora de hidrocarburos, junto con otras Formaciones como La Casita, La Peña y Olmos. Estas formaciones están conformadas por secuencias de rocas que se depositaron en ambientes marinos con aporte de terrígenos y materia orgánica tipo II y predominantemente tipo III dentro de la Cuenca de Sabinas (Piedad-Sánchez, 2004; Martínez et al., 2015), variando el poder reflector de la vitrinita que alcanza hasta $\mathrm{Ro}=2.7 \%$ en las rocas más profundas de la formación La Casita (Piedad-Sánchez, 2004; Martínez et al., 2015). Eguíluz de Antuñano (2001) propone un modelo térmico generalizado para la Cuenca de Sabinas con base en la profundidad y la edad de las Formaciones (M.a.), en el que se plantea que la Formación Eagle Ford en conjunto con la Formación Austin se encuentran en la fase principal de la generación de gas (Figura 11). Este modelo concuerda con lo expuesto por Piedad-Sánchez (2004) al analizar la historia de sepultamiento, y, con ello, precisa con algunos
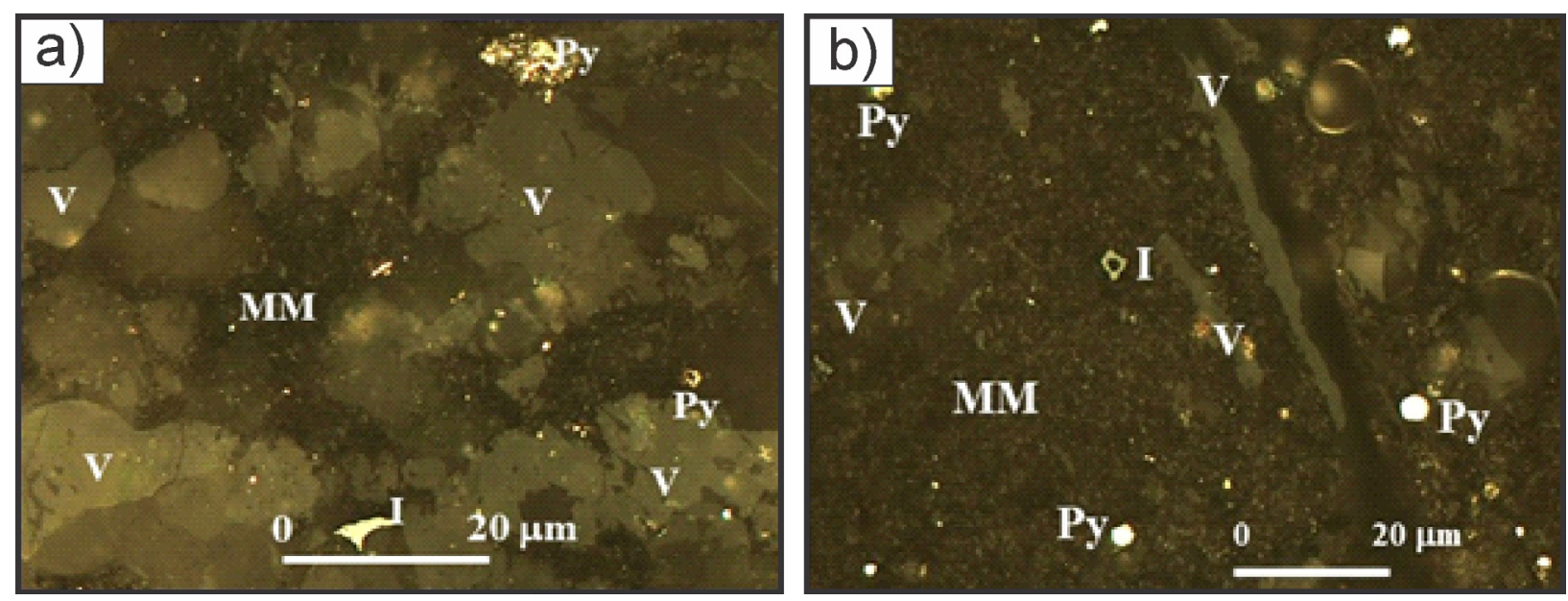

Figura 9 Microfotografías de petrografía orgánica de lutitas de la Formación Eagle Ford del área de Nueva Rosita, Coahuila, con presencia de vitrinita (V) con un \%Ro entre 0.67 y 1.29 , inertinita (I) y una matriz arcillosa con pirita (Py). 
datos medidos del poder reflector de la vitrinita $\%$ Ro, que varían de 0.67 a $1.29 \%$ en muestras de pozos petroleros de PEMEX (Figura 9) en el área de Monclova- Nueva Rosita, Coahuila (Piedad-Sánchez, 2004).

La maduración térmica de la Formación Eagle Ford, medida con el poder reflector de la vitrinita en muestras de pozos petroleros, indica también una tendencia diferencial donde se tienen algunos resultados de $\%$ Ro $=0.6 \%$ a 1,380 metros de profundidad en la zona de Progreso, Coahuila, y $1.88 \%$ a 660 metros en la zona de Barroterán, Coahuila (Piedad-Sánchez, 2004). No obstante, los resultados de $\%$ Ro de 0.60 a 1.88 en el interior de la Cuenca de Sabinas (Piedad-Sánchez, 2004; Petróleos Mexicanos, 2011, 2012) coindicen con la variabilidad térmica mostrada por los datos de Th (Figura 10), sugiriendo así que la Cuenca de Sabinas mostrará un potencial de gas y aceite en función de la evolución geológica local impactada por su paleogeografía, la interacción con fluidos y su deformación laramídica.

Respecto a los análisis microtermométricos, tenemos que la materia orgánica maduró de manera diferencial a lo largo de la paleogeografia, presentando las temperaturas más bajas al norte de la
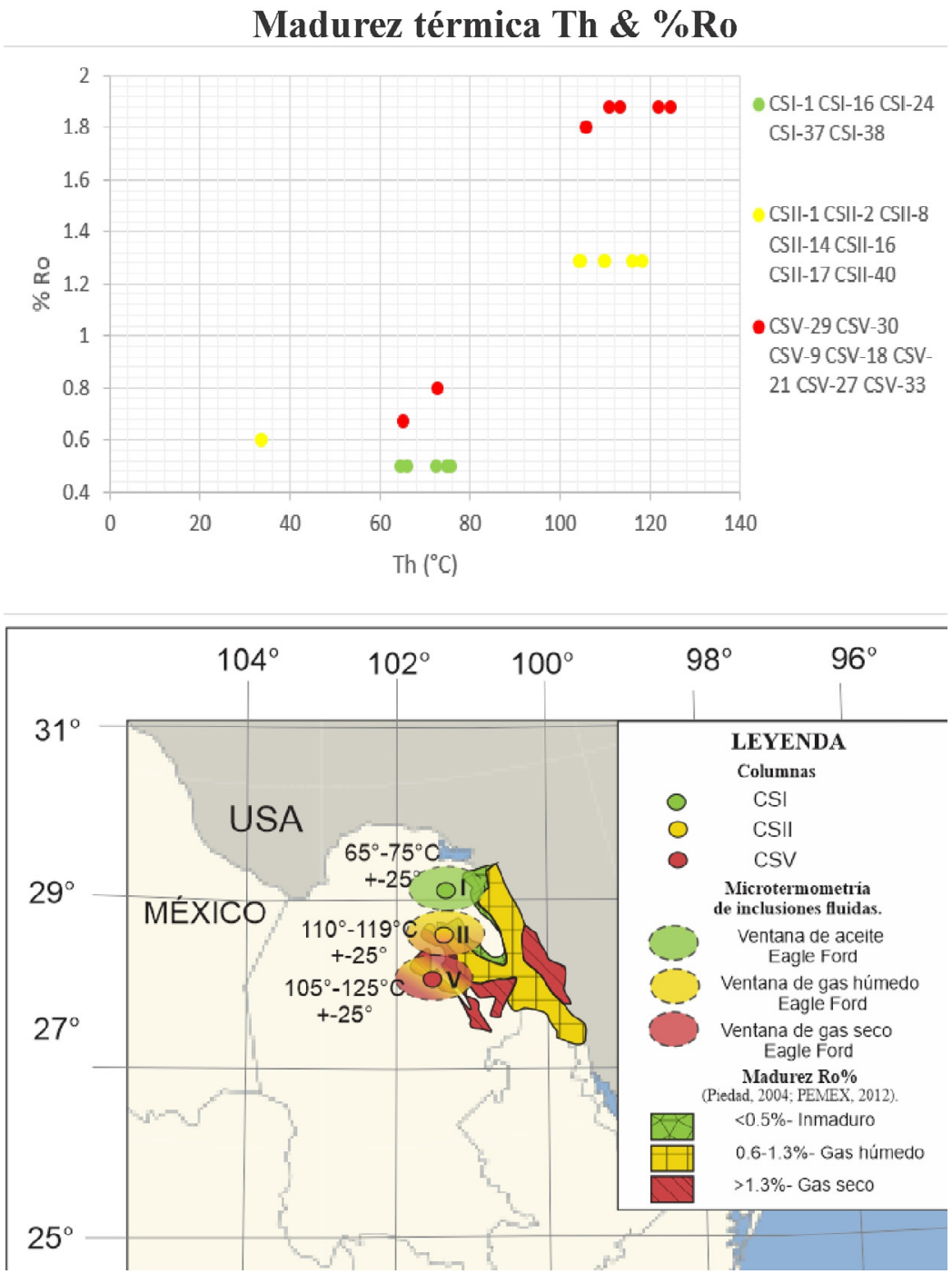

Figura 10 Diagrama de Th \& \%Ro, en el que se relacionan los datos para la determinación de la madurez orgánica de la Formación Eagle Ford. En la parte inferior se observa la ubicación de las columnas estratigráficas con el grado de madurez térmico observado en este análisis microtermométrico, así como también el grado de madurez \%Ro aproximado, tomando en cuenta los datos de PEMEX (2012) y Piedad (2004). 
Península Burro-peyotes (columna I) y las más elevadas en el interior de la Cuenca de Sabinas (columna V). Siendo la columna I la única que solo maduró dentro de la ventana de generación de aceite, mientras que las columnas II y V si alcanzaron a entrar a la ventana de generación de gas en base a la temperatura de homogeneización promedio (temperatura mínima). Además, en la columna $\mathrm{V}$, dentro de la Cuenca de Sabinas, existe también una variación a lo largo de la misma secuencia, observando que a la base se obtienen las temperaturas más altas y disminuyen hacia la cima, por lo que podemos decir que la maduración de la materia orgánica tampoco fue homogénea verticalmente dentro de la misma secuencia (Figura 7), misma tendencia diferencial reportada por Piedad- Sánchez (2004) en la zona de
Barroterán, Coahuila. También se presentan algunos valores de temperaturas anormales muy bajas que en realidad deben corresponder a cementos formados durante la exhumación.

Con los datos microtermométricos obtenidos, los valores de \%Ro y con base en el diagrama de Boyer et al., (2007) (Figura 11), se tiene que la Formación Eagle Ford, de manera general, se encuentra en la fase de generación de aceite hacia la plataforma Burro-Peyotes y alcanza a entrar en la fase principal de la generación de gas en el centro de la Cuenca de Sabinas, presentando una maduración diferencial. Según Eguíluz de Antuñano et al., (2001) esta formación, en general, alcanza las condiciones necesarias para la generación de gas durante el Paleoceno. En este trabajo,

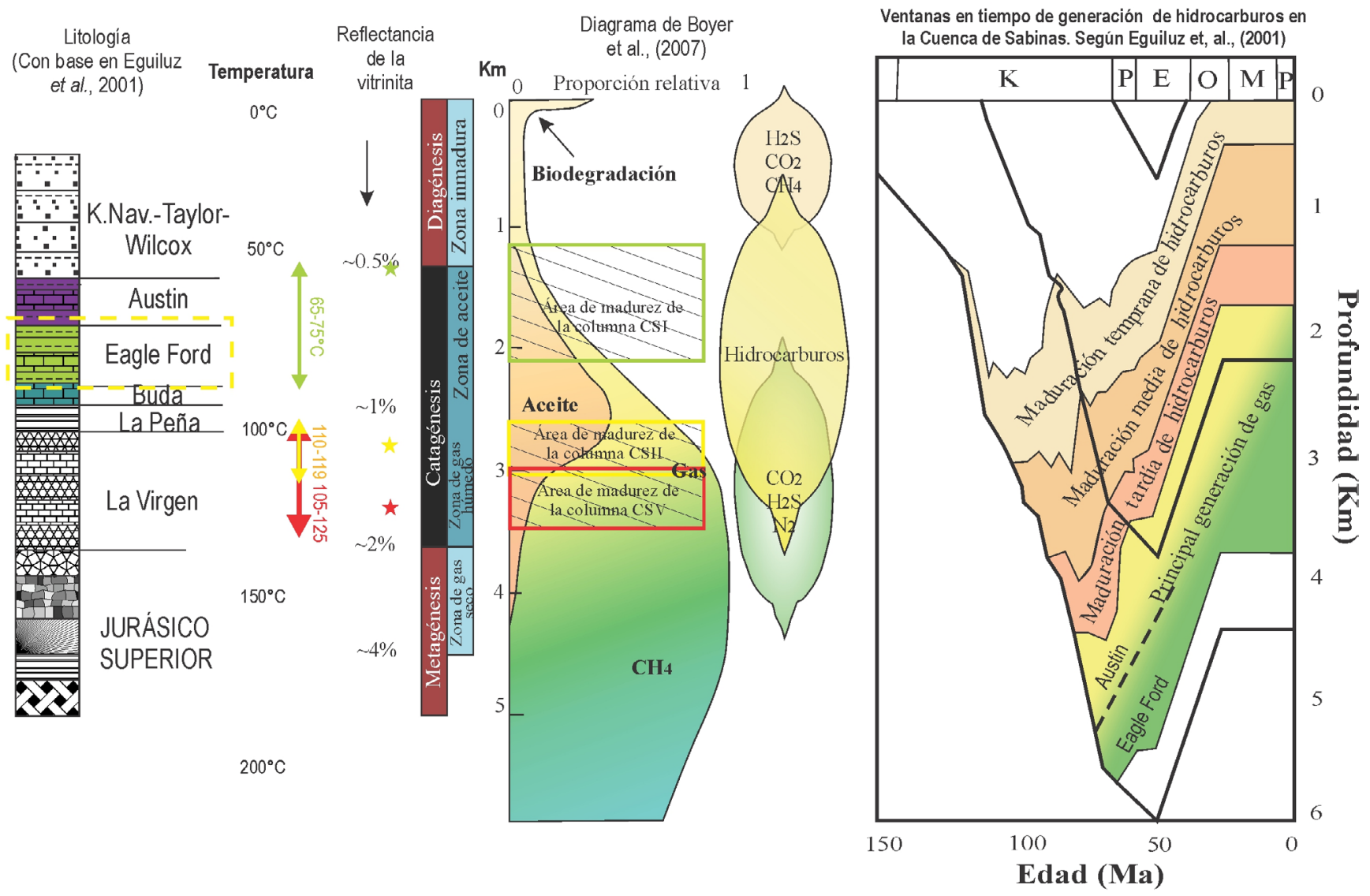

Figura 11 Diagrama modificado de Boyer et al., 2007, donde se muestra la rango de madurez térmica correspondiente a la Formación Eagle Ford en el Norte de Coahuila, y el modelo propuesto por Eguiluz de Antuñano (2001).Se desprende que de manera general, la columna I se localiza en la zona de generación de aceite y las columnas II y V en la zona de generación de gas húmedo. Las flechas indican en rango de temperaturas alcanzadas en el análisis de inclusiones fluidas, mientras que las estrellas indican datos de \%Ro cerca de las zonas donde se tomaron cada una de las columnas. 
las temperaturas de homogeneización obtenidas a partir del análisis de inclusiones fluidas de la Cuenca de Sabinas representan las temperaturas mínimas de atrapamiento de los fluidos, las salinidades determinadas representan el equivalente total de sales disueltas (Wilkinson, 2001).

En este último caso solo se puede saber si los fluidos son predominantemente ricos en $\mathrm{NaCl}$ (de acuerdo con su Tff) y el porcentaje del poder reflector de la vitrinita representa la temperatura máxima a la que estuvo sometida la materia orgánica. El kerógeno tipo II normalmente está relacionado con la materia orgánica marina depositada en un ambiente reductor y, según Santamaría-Orozco et al., (2009), se caracteriza por que sus organismos son de origen marino y por su textura sapropélica con escasos aportes de material terrígeno, depositados mayoritariamente en ambientes de plataforma continental constituido por alginitas, esporinitas, cutinitas, polen y exinitas. En etapas tempranas de madurez, este tipo de kerógeno comienza a generar aceites parafínicos pobres en ceras y ricos en naftenos, mientras que en etapas avanzadas de madurez genera aceites ricos en aromáticos y mayores cantidades de gases (Figura 11), lo que se espera para la Formación Eagle Ford. Por otro lado, las temperaturas de los análisis microtermométricos en la Cuenca de Sabinas publicados por González-Partida et al., (2008a, 2008b) y González-Sánchez et al., $(2007,2009)$ para yacimientos del tipo MVT (Figura 8) son el resultado de una evolución térmica dinámica, influenciada por el espesor de sepultamiento, el gradiente geotérmico y geobárico y la interacción de fluidos calientes profundos provenientes del interior de la cuenca, así como fluidos meteóricos u otras salmueras, mientras que para la Formación Eagle Ford tenemos una maduración térmica estática originada, principalmente, por la profundización de la secuencia que iba generando un calor interno en la roca $\left(25^{\circ}\right.$ a $\left.30^{\circ} / \mathrm{Km}\right)$ y, por ende, la maduración paulatina del kerógeno a gas. Esta maduración térmica debió estar también condicionada por los movimientos compresionales de la Orogenia Laramide. Para el caso de la península Burro-Peyotes la deformación ocasionada por la Orogenia Laramide no fue tan intensa, por lo que los espesores fueron los determinantes para que las lutitas alcanzaran las temperaturas que observamos en el análisis microtermométrico de inclusiones fluidas.

\section{Conclusiones}

La Formación Eagle Ford está compuesta por lutitas calcáreas con un alto contenido de materia orgánica del tipo II y III (0.5 a 8\% de COT), intercalada con calizas arcillosas, clásticas o bioclásticas, siendo así una formación heterogénea y anisotrópica por sus cambios litológicos perpendiculares (estratificación) y paralelos (cambios de facies).

Esta secuencia se depositó en una plataforma somera protegida por bancos de arenas carbonatados con baja oxigenación o condiciones anóxicas, afectada esporádicamente por un fuerte oleaje o por tormentas. Esto influye en el contenido de materia orgánica.

En conjunto; las observaciones petrográficas, las temperaturas de homogeneización y el poder reflector de la vitrinita indican que la madurez térmica que alcanzó la materia orgánica de la Formación Eagle Ford pertenece a la etapa catagenética. La maduración de la materia orgánica fue el resultado de un incremento de temperatura por el sepultamiento de la formación y con una baja interacción de salmueras a través del tiempo, lo que ocasionó la diagénesis dentro de un sistema estático y, por ende, la maduración paulatina del kerógeno. La maduración térmica estuvo condicionada por los movimientos compresionales de la Orogenia Laramide, que sepultaron o expusieron partes de la Formación Eagle Ford en el interior de la cuenca, creando la variación de temperatura que observamos a lo largo de la paleogeografía.

Finalmente, las columnas CSII y CSV, con valores de COT mayor al 2\%, un poder reflector de la vitrinita $\%$ Ro entre 1.29 y 1.88 , y Th entre $105^{\circ}$ y $125^{\circ} \mathrm{C}$, alcanzaron las condiciones necesarias para la generación de gas húmedo.

Aunque se requieren más estudios complementarios, la Formación Eagle Ford cumple con 
algunos requisitos para ser considerada como un recurso potencial de gas de tipo no convencional, como: espesores entre 90 y 125 m, una gran extensión, desarrollo de porosidad secundaria (fracturas y porosidad inter e intragranular), heterogeneidad litológica, presencia de kerógeno II y III, COT $>2 \%, \%$ Ro $>1.2$, Th dentro de la etapa catagenética y profundidades de hasta casi $4 \mathrm{~km}$.

\section{Agradecimientos}

El presente trabajo corresponde a estudios realizados por González Betancourt Aurea Yahaira para poder obtener el grado de Doctorado en el Centro de Goeciencias UNAM, Campus Juriquilla, donde Vázquez Ramírez Juan Tomas apoyó en la preparación de muestras, y con quien quedamos ampliamente agradecidos. Igualmente, queremos agradecer a los evaluadores: el Dr. Demetrio Santamaría-Orozco y la Dr. Mariene Gutiérrez Neri por sus valiosas observaciones y al editor en jefe Dr. Antoni Camprubí. Gracias también al CONAGyT por el soporte financiero de la beca.

\section{Referencias}

Alvarado-Ortega J., Blanco-Piñón A., y PorrasMúzquiz H., 2006, Primer registro de Sarodon (Teleostei: Ichthyodectiformes) en la cantera La Mula, Formación Eagle Ford (Cretácico Superior: Turoniano), Múzquiz, Estado de Coahuila, México: Revista Mexicana de Ciencias Geológicas, 23 (2), 107-112.

Askenazi, A., Biscayart, P., Cáneva, M., Montenegro, S., Moreno, Marcos., 2013, Analogía entre Formación Vaca Muestra y shale gas/oilplays de EEUU: Society of Petroleum Engineers. 2-8p. http://www. spe.org.ar/locker/pdf/SPE_JJPP0003.pdf

Barboza, L.D., Santiago C.B., Izaguirre R.M.A., Martínez R.C., Gracia V.M., 1997, Servicio Geológico Mexicano. Carta
Geológico-Minera Monclova, G14-4, Escala 1:250,000 Estados de Coahuila y Nuevo León, Informe técnico, inédito.

Bathurst, R.G.C., 1975, Carbonate sediments and their diagenesis: Amsterdam, Holanda, Elsevier, 660 p.

Berner R. A., 1980, Early Diagenesis-a theoretical approach: Princeton University Press. 241 p. https://doi.org/10.2307/j.ctvx8b6p2

Boiron, M.C., Dubessy,J., 1994, Determination of fluid inclusion composition: microanalytical techniques, en Fluid inclusions in minerals: methods and applications, De Vivo, B., Frezzotti, M.L. (Eds.): Pontignano- Siena, Italia, short course "Inclusions in Minerals", 1-4 septiembre, 45-71p.

Boyer, C., Kieschnick, J., Suárez-Rivera, R., Lewis, R.E., and Waters, G., 2007, Producción de gas desde su origen: Oilfield Review, 36-49 p.

Budd, D.A., Saller, A.H., Harris, P.M., 1995, Unconformity and porosity in carbonate strata: American Association of Petroleum Geologists Memoir, 63, 313. https://doi. org/10.1306/M63592

Buschaert, S., Fourcade S., Cathelineaux M., Deloule E., Martineau F., Ayt Ougougdall M., \& Trouiller, A., 2004, Widespread cementationinduced byinflow of continental water in the eastern part of the Paris basin: $\mathrm{O}$ and $\mathrm{C}$ isotopic study of carbonate cements: Applied Geeochemistry 19(8), 1201-1215. https://doi.org/10.1016/j. apgeochem.2003.01.001

Chillingarian, G.V., Mazullo, S.J., Rieke, H.H. (eds.), 1992, Carbonate reservoir characterization: a geologic-engineering analysis, part I-II: Amsterdam, Holanda, Elsevier, 639 p.

Cobban, W.A., 1988, Tarrantoceras Stephenson and related ammonoid genera from Cenomanian (Upper Cretaceous) rocks in Texas and the Western Interior of the United States: U.S. Geological Survey Professional Paper, 1473, 51 p. https:// pubs.usgs.gov/pp/1473/report.pdf 
Corona-Esquivel, R., Tritlla, J., BenavidesMuñoz, M.E., Piedad-Sánchez, N., Ferrusquía- Villafranca, I., 2006, Geología, estructura y composición de los principales yacimientos de carbón mineral en México: Boletín de la Sociedad Geológica Mexicana, 58(1),141-160. https://doi.org/10.18268/ bsgm2006v58n1a5

Eguíluz de Antuñano, S.,1984, Tectónica Cenozoica del Norte de México: Boletín de la Asociación Mexicana de Geólogos Petroleros 36(1), 43-62.

Eguíluz, de Antuñano, S., 2001, Geologic Evolution and Gas Resources of the Sabinas Basin in Northeastern México, in: Bartolini, C., Buffler, R. T., and Cantú-Chapa, A., eds., The western Gulf of México Basin: Tectonics, sedimentary basins, and petroleum systems: American Association of Petroleum Geologists Memoir 75, 241270. https://doi.org/10.1306/M75768

Eguíluz de Antuñano, S., 2007, Exploración petrolera cuantitativa, ejemplos y resultados de los estudios de plays en la Cuenca de Sabinas Coahuila, México: GeoCiencia. Revista del Servicio Geologico Mexicano 1(1), 9-36. http://www.sgm.gob.mx/pdfs/ geociencia/ABRIL_mini.pdf

Enciso-Cardenas, J.J., 2015, Estudio de las propiedades de adsorción - desorción de gases en los sistemas petroleros no convencionales en Mexico y su aplicación al modelo cinético de generacion de hidrocarburos: Université de Lorraine, 2-452 p. https://hal.univ-lorraine.fr/ tel-01754483/document

Escalante M.J.C., Rocha, R. M., Chiapa, G.R.R., Bastida, J.R., 2002, Servicio Geológico Mexicano, Carta Geológico-Minera Ciudad Acuña, H14-7, Escala 1:250,000 Estado de Coahuila: Informe técnico, inédito.

Ewing, T.E., 2003, Review of the tectonic history of the Lower Rio Grande border region, South Texas and Mexico, and implications for hydrocarbon exploration: Society of
Independent Professional Earth Scientists, SIPES Newsletter 40 (2), 16-21.

Flügel, E., 2004, Microfacies of carbonate rocks. Analysis, interpretation, and application: Berlin - Heidelberg, Alemania, Springer, p. 976. https://doi. org/10.1007/978-3-662-08726-8

Goldhammer, R. K., Lehmann, P. J., Todd, R. G., Wilson, J. L., Ward, W. C., and Johnson, C. R., 1991, Sequence stratigraphy and cyclostratigraphy of the Mesozoic of the Sierra Madre Oriental, northeast Mexico, a field guide-book. Gulf Coast Section: Society of Economic Paleontologists and Mineralogists, $85 \mathrm{p}$.

Goldhammer, R. K., Dunn, P. A., and Lehmann, P. J., 1993, The origin of high- frequency platform carbonate cycles and third-order sequences (Lower Ordovician El Paso Group, west Texas): Constraints from outcrop data, inverse and forward stratigraphic modeling: Journal of Sedimentary Petrology 63, 318-359. https://doi.org/10.1306/ d4267afa-2b26-11d7-8648000102c1865d

Goldhammer, R. K., 1999, Mesozoic sequence stratigraphy and paleogeographic evolution of northeast of Mexico, in Bartolini, C., Wilson, J.L., and Lawton, T.F., eds., Mesozoic Sedimentary and Tectonic History of North-Central Mexico. Boulder, Colorado: Geological Society of North America Special Paper 340, 1-58p.

Goldhammer, R. K., and Johnson, C. A., 2001, Middle Jurassic-Upper Cretaceous Paleogeographic evolution and sequencestratigraphic framework of the northwest Gulf of México rim, in: Bartolini, C., Buffler, R. T., and Cantú-Chapa, A., eds., The western Gulf of México Basin: Tectonics, sedimentary basins, and petroleum systems: American Association of Petroleum Geologists Memoir 75, p. 4581. https://doi.org/10.1306/M75768

Goldstein, R.H., 2003, Petrographic analysis of fluid inclusions, in: Iain Samson, Alan 
Anderson, Dan Marshall, eds., Fluid Inclusions: Analysis and Interpretation: Mineralogical Association of Canada, V. 32, 9-53p.

Goldstein, R.H., y Reynolds, T.J., 1994, Systematics of fluid inclusions in diagenetic minerals: SEPM Society for Sedimentary Geology 31, 1-199. https://doi. org/10.2110/scn.94.31

González-Betancourt, A.Y., 2018, Estudio diagenético de la Formación Eagle Ford (Cenomaniano tardío. Turoniano) como productora de gas no convencional: México, Universidad Nacional Autónoma de México, Tesis de Maestría, 358 p.

González, G.R. y N.Q. Holguín, 1992, Las rocas generadoras de México: Boletín de la Sociedad Mexicana de Geólogos Petroleros 42(1), 16-30.

González-Partida, E., Camprubí, A., Canet, C., González-Sánchez, F., 2008a. Fisicoquímica de salmueras e hidrocarburos en cuencas petroleras y en depósitos minerales tipo Mississippi Valley y asociados primera parte: temperatura, presión y composición de inclusiones fluidas: Boletín de la Sociedad Geológica Mexicana 60 (1), 11-22. https:// doi.org/10.18268/bsgm2008v60n1a2

González-Partida, E., Camprubí, A., Canet, C., González-Sánchez, F., 2008b. Fisicoquímica de salmueras e hidrocarburos en cuencas petroleras y en depósitos minerales tipo Mississippi Valley y asociados. Segunda parte. Ejemplos de la cuenca de Sabinas y la cuenca del Sureste, México. Boletín de la Sociedad Geológica Mexicana 60 (1), 23 - 42. https://doi.org/10.18268/ bsgm2008v60n la3

González-Ruiz, L. E., González-Partida E., Martínez, L., Pironon, J., Camprubí, A., Vega-González, M., 2015, Fenómenos diagenéticos en calizas del Jurásico-Cretácico de un sector de las cuencas de HuimanguilloComalcalco-Alto de Jalpan y primeras observaciones mediante microscopio electrónico de barrido y microtomografía 3D:

Boletín de la Sociedad Geológica Mexicana 67(3), 517-531. https://doi.org/10.18268/ bsgm2015v67n3a14

González-Sánchez, F., Puente-Solís, R., Gónzalez-Partida, E., Camprubí, A., 2007, Estratigrafía del Noreste de México y su relacion con los yacimientos estratoligados de fluorita, barita, celestina y $\mathrm{Zn}-\mathrm{Pb}$ : Boletín de la Sociedad Geologica Mexicana 59(1), 43-62. https://doi.org/10.18268/ bsgm2007v59n1a4

González-Sánchez, F., Camprubí, A., GonzálezPartida, E., Puente-Solís, R., Canet, C., Centeno-García, E., and Atudorei, V., 2009, Regional stratigraphy and distribution of epigenetic stratabound celestine, fluorite, barite and $\mathrm{Pb}-\mathrm{Zn}$ deposits in the MVT province of northeastern Mexico: Mineralium Deposita 44(3), 343-361. https://doi.org/10.1007/ s00126-008-0212-4

Harries, P.J., Kauffman E.G., Crampton, J.S., Bengston, P., Chech S., Crame J.A., Dhondt A.V., Ernst G., Hilbrecht, H., López G., Mortimere R., Tröger K.A., Walaszcyk I., Wood C.J., 1996, Lower Turonian Euramerican Inoceramidae: A morphologic, taxonomic, and bioestratigraphic overview: In: New developments in Cretaceous research topics. Proceedings of the 4th International Cretaceous Symposium, Hamburg, 1992. Hamburg, Universitat Hamburg, 641-671.

Horsfield, B., Rullkötter, J., 1994, Diagenesis, catagenesis, and metagenesis of organic matter, en Magoon, L.B., Dow, W.G., The petroleum system-from source to trap: American Association of Petroleum Geologists Memoir, 60, 189-199.

Longoria, FJ., 1984. Stratigraphic studies in the Jurassic of North-eastern Mexico: Evidence for the origin of Sabinas basin, in the Jurassic of the Gulf Rim: Gulf Coast Section, Society for Sedimentary Geology 
(SEPM) Foundation. Third Annual Research Conference Proceeding, 171-193. https://doi.org/10.5724/gcs.84.03.0171

Márquez-Domínguez, B., 1979. Evaluación petrolera de sedimentos carbonatados del Cretácico en el Golfo de Sabinas, NE de México. XVII Consejo Anual de la AIPM, Cancún, Quintana Roo, México, 28-37.

Martínez R. L., Miranda H. A., Sánchez G. E., Pérez V.M.A., 2000, Servicio Geológico Mexicano, Carta Geológico-Minera Nueva Rosita, G14-1, Escala 1: 250,000 Estados de Coahuila y Nuevo León. Informe técnico.

Martínez, L., Camacho, L.F., Piedad-Sanchez, N., González-Partida, E., Suárez- Ruiz, I., Enciso, J., 2015, Entorno diagenético en el Bloque Pirineo, Cuenca de Sabinas, México: Interacción agua-roca-hidrocarburo: RIIIT Revista Internacional de Investigación e Innovación Tecnológica 13, 2-32.

Martínez-Contreras,J. F., 2015, Estudio estratigráfico- geoquímico en petróleo y gas de lutitas de la Formación Eagle Ford, noroeste de Villa Hidalgo, estado de Coahuila, noreste de Mexico: México, Universidad Nacional Autónoma de México, Tesis de Maestría, $151 \mathrm{p}$.

Molina, E. , 2004, Micropaleontología: España, Prensas Universitarias de Zaragoza.

Morad, S., KetzerJ. M, De Ros L. F., 2000, Spatial and temporal distribution of diagenetic alteration in siliciclastic rochs- implications for mass transfer in sedimentary basin: Sedimentology 47, 955- 120. https://doi. org/10.1046/j.1365-3091.2000.00007.x

Moreman, W.L.,1927, Fossil Zones of the Eagle Ford of North Texas: Journal of Paleontology, 1(1), 89-101.

Morton, J. P., 1985, Rb-Sr evidence for punctuated illite/smectite diagenesis in the Oligocene Frio Formation Texas Gulf Coast: Bulletin Geological Society of America 96(1), 114-122. https://doi. org/10.1130/0016-7606(1985)96<114:RE FPID $>2.0 . \mathrm{CO} ; 2$
Padilla y Sánchez, R.J., 1986, Post- Paleozoico tectonics of Northeast Méxicoand its role in the evolution of the Gulf of Mexico: Geofísica International 25(1), 157-206.

Padilla y Sánchez, R.J., 2016, Late Triassic- Late Cretaceous Paleogeography of Mexico and the Gulf of México: Gulf Coast Association of Geological Societies Foundation.

Pagel, M., Barbarand,J., Beaufort, D., Gautheron, C., Pironon, J., 2014, Bassins sédimentaires: Les marqueurs de leur histoire thermique: Société Géologique de France (SGF), Géospheres Collection. 227 p.

Pérez de la G. J.A., Arzabala M. J., Tarín Z. G., De los Santos M. J., 2000, Servicio Geológico Mexicano, Geológico-Minera Ocampo, G13-3, Escala 1: 250000 Estado de Coahuila y Chihuahua. Informe técnico.

Petróleos Mexicanos, 1988, Estratigrafía de la República Mexicana:Mesozoico, Subdirección de Producción Primaria, Coordinación Ejecutiva de Exploración, 229 p.

Petróleos Mexicanos, 2011, Las reservas de hidrocarburos de México: México, D.F., Pemex Exploración y producción, 121 p.

Petróleos Mexicanos, 2012, Situación Actual y Perspectivas de Pemex, en Expo foro 2013, México, D.F., 1-8.

Piédad-Sánchez, N., 2004, Prospection des hydrocarbures par une approche intégrée de pétrographie, géochimie et modélisation de la transformation de la matière organique: Analyse et reconstitution de l'histoire thermique des Bassins Carbonifére Central des Asturies (Espagne) et Sabinas-Piedras Negras (Coahuila, Mexique) La Cuenca de Sabinas (Coahuila México): Université de Lorraine, Francia. Tesis de Doctorado. 356, p.

Piedad-Sánchez, N., 2005, Estudio de la Industria del Carbón en la Región Carbonífera del Estado de Coahuila y del cluster del carbón a nivel mundial: Corporación Mexicana de Investigación en Materiales, S.A., 16-29 p.

Pindell, J. L., 1985, Alleghenian reconstruction and subsecuent evolution of the Gulf of 
Mexico, Bahamas, and Proto-Caribbean: Tectonics 4(1), 1-39. https://doi. org/10.1029/TC004i001p00001

Pindell, J. L., 1993, Regional synopsis of the Gulf of Mexico and Caribbean evolution, in: Pindell, J.L., and Perkins, B.F., eds., Mesozoic and early Cenozoic development of the Gulf of Mexico and Caribbean region. Gulf Coast Section, SEPM (Society for Sedimentary Geology), Foundation, 13th Annual Research Conference, 251-274 p. https://doi.org/10.5724/gcs.92.13.0251

Pindell, J. L., and Barrett, S. F., 1991, Geological evolution of the Caribbean region; A platetectonic perspective: The Caribbean Region, Gabriel Dengo, J.E. Case. The Geological Society of America, p. 405-432. https:// doi.org/10.1130/DNAG-GNA-H.405

Pindell, J. L., Cande, S. G., Pitman, W. C., Rowley, D. B., Dewey, J. F., Labrecque, J., Haxby, W., 1988, A plate-kinematic framework for models of Caribbean evolution: Tectonophysics 155, 121-138. https://doi. org/10.1016/0040-1951(88)90262-4

Pindell, J.L., and Kennan, L., 2009, Tectonic evolution of the Gulf of México, Caribbean and northern South America in the mantle reference frame: an update: Geologica Society 328(1), 1-55. https:// doi.org/10.1144/sp328.1

Robeck, R.C., Pesquera, R.V., Ulloa, S.A., 1960, Geología y depósitosde carbón en la región de Sabinas, estado de Coahuila: Sociedad Geológica Mexicana, libreto de guía de la excursión C-3 del XX Congreso Geológico Internacional, 87-115 p.

Roedder, E., 1984, Fluid Inclusions: Reviews in Mineralogy, Mineralogical Society of America 12, p. 646.

Roemer (Römer), F.,1852, Die Kreidebildungen von Texas, und ihre organischen Einschlüsse: il. Bonn, Adolph Marcus, pp.100, 10 pls. https://doi.org/10.5962/bhl.title.15015

Robinson, G.R., 1997, Hydrocarbon source rock variability within the Austin Chalk and Eagle Ford Shale (Upper Cretaceous), East
Texas, U.S.A: International Journal of Coal Geology 34(3-4), 287-305. https://doi. org/10.1016/S0166-5162(97)00027-X

Romo, R. J.R., Herrera M. J., Rodríguez R.J.S., Larrañaga O. G., 2002, Servicio Geológico Mexicano, Carta Geológico-Minera San Miguel, H13-12, Escala 1:250,000, Estados de Coahuila y Chihuahua Informe técnico.

Salvador, A., 1987, Late Triassic-Jurassic paleogeography and origin of Gulf of Mexico basin: American Association of Petroleum Geologists Bulletin 71(4), 419451. https://doi.org/10.1306/94886EC51704-11D7-8645000102C1865D

Salvador, A., 1991a. The Gulf of Mexico basin: Boulder, Colorado: Geological Society of America, Geology of North America J, 568 p. Salvador, A., 1991b, Triassic-Jurassic: in Salvador, A., ed., The Gulf of Mexico basin: Boulder, Colorado: Geological Society of America, Geology of North America J, 131-180 p.

Salvador, A., 1991c, Origin and development of the Gulf of Mexico basin: in Salvador, A., ed., The Gulf of Mexico basin: Boulder, Colorado: Geological Society of America, Geology of North America J, 389-444 p.

Sánchez, B., G., Jiménez, H. A., Caballero M.J.A., 2002, Servicio Geológico Mexicano, Carta Geológico-Minera Manuel Benavides, H139, Escala 1:250,000 Estados de Coahuila y Chihuahua. Informe técnico, inédito.

Santamaría-Orozco, D., Arenas, P. R., Escamilla, H., 1990, Normalización de la Nomenclatura Estratigráfica en las Cuencas Mesozoicas de México (Etapa I: Zona Norte. Cretácico). Instituto Mexicano Petróleo. Proyecto CAO-3052. 140 p.

Santamaría-Orozco. D., Ortuño, A. F., Adatte, T., Ortíz, U. A., Riba, R. A., Franco, N.S., 1991, Evolución geodinámica de la Cuenca de Sabinas y sus implicaciones petroleras, Estado de Coahuila: Instituto Mexicano del Petróleo, internal report, pp. 209.

Santamaría-Orozco, D., 2000, Organic geochemistry of Tithonian source rocks and associated oils from the Sonda de 
Campeche, México: Aachen, Alemania, Tesis doctoral inédita, Technical University RWTH 168 p.

Santamaría-Orozco, D., Horsfield, B., 2003, Gas generation potential of Upper Jurassic (Tithonian) Source Rocks in the Sonda de Campeche, México: AAPG Memoir, 79, 349-363.

Santamaría-Orozco, D.M., Amezcua Allieri, M. A., Carrillo Hernández, T. J., 2009, Generación de petróleo mediante experimentos de pirólisis: revisión sobre el conocimiento actual. Boletín de la Sociedad GeolóGica Mexicana 61(3), 2009, 353-366. https://doi.org/10.18268/ bsgm2009v61n3a5

Santiago, C.B., Martínez R. C.J, Sánchez B. G., Chiapa G. R., Palacios G.R., 2000, Servicio Geológico Mexicano, Carta GeológicoMinera Monterrey, G14-7, Escala 1:250,000, Estados de Coahuila, Nuevo León y Zacatecas, Informe técnico, inédito. Santiago, C. B., Herrera M.J.C., Ontiveros E. E., Martínez R.L., 2003, Servicio Geológico Mexicano, Carta Geológico-Minera Piedras Negras, H14-10, Escala 1:250,000 Estado de Coahuila yChihuahua. Informe técnico.
Sellards, E.H., Adkins W.S., F.B. Plummer, 1966, The Geology of Texas vol. I Stratigraphy: The University of Texas, Austin, Bureau of Economic Geology, UT Bulletin 3232, 1,107 p.

Shumard, B. F., 1860, Descriptions of new Cretaceous fossils from Texas, Ac. Sc. St. L., Tr., 1:590-610.

Wilkinson, J.J., 2001, Fluid Inclusions in Hydrothermal Ore Deposits: Lithos 55(14), 229-272. https://doi.org/10.1016/ s0024-4937(00)00047-5

Wilson, J.L., 1990, Basement structural controls on Mesozoico carbonates facies in northeastern, México: A review, en Tucker, M.E., Wilson, J.L., Crevello, P.D., Sarg, J.R., Read,J.F. (Eds.), Carbonate platforms, facies, sequences and evolution: International Association of Sedimentologists, Special Publication, 9, 235-255. https://doi. org/10.1002/9781444303834.ch9

Worden, R. H., Burley S. D., 2003, Sanndstone diagenesis: the evolution of sand to stone.In Sanston diagenesis: Recent and ancient, Burley., S.D., Worden, R. H., (Eds.): International Association of Sedimentologists, 3-44 p. https://doi. org/10.1002/9781444304459.ch 